\title{
Understanding B-type supergiants in the low metallicity environment of the SMC
}

\author{
C. Trundle ${ }^{1,2}$, D. J. Lennon ${ }^{1}$, J. Puls ${ }^{3}$, and P. L. Dufton ${ }^{2}$ \\ 1 The Isaac Newton Group of Telescopes, Apartado de Correos 321, 38700, Santa Cruz de La Palma, Canary Islands, Spain \\ 2 The Department of Pure and Applied Physics, The Queen's University of Belfast, Belfast BT7 1NN, Northern Ireland \\ ${ }^{3}$ Universitäts-Sternwarte München, Scheinstr. 1, 81679, Germany
}

Received 16 September 2003 / Accepted 5 December 2003

\begin{abstract}
Spectroscopic analyses of 7 SMC B-type supergiants and 1 giant have been undertaken using high resolution optical data obtained on the VLT with UVES. FASTWIND, a non-LTE, spherical, line-blanketed model atmosphere code was used to derive atmospheric and wind parameters of these stars as well as their absolute abundances. Mass-loss rates, derived from $\mathrm{H}_{\alpha}$ profiles, are in poor agreement with metallicity dependent theoretical predictions. Indeed the wind-momenta of the SMC stars appear to be in good agreement with the wind-momentum luminosity relationship (WLR) of Galactic B-type stars, a puzzling result given that line-driven wind theory predicts a metallicity dependence. However the galactic stars were analysed using unblanketed model atmospheres which may mask any dependence on metallicity. A mean nitrogen enhancement of a factor of 14 is observed in the supergiants whilst only an enrichment of a factor of 4 is present in the giant, AV216. Similar excesses in nitrogen are observed in O-type dwarfs and supergiants in the same mass range, suggesting that the additional nitrogen is produced while the stars are still on the main-sequence. These nitrogen enrichments can be reproduced by current stellar evolution models, which include rotationally induced mixing, only if large initial rotational velocities of $300 \mathrm{~km} \mathrm{~s}^{-1}$ are invoked. Such large rotational velocities appear to be inconsistent with observed $v \sin i$ distributions for O-type stars and B-type supergiants. Hence it is suggested that the currently available stellar evolution models require more efficient mixing for lower rotational velocities.
\end{abstract}

Key words. stars: atmospheres - stars: early-type - stars: supergiants - stars: mass-loss - stars: abundances - stars: evolution

\section{Introduction}

Due to its low metal content and close proximity the Small Magellanic Cloud (SMC) is a key laboratory in which to study massive stars. Its metallicity $(Z)$, or more accurately the abundance of its iron peak and alpha-processed elements, is approximately one fifth solar, making it an excellent template for studies of metal poor starbursts and high redshift star-forming galaxies (Leitherer et al. 2001; Pettini et al. 2000). Its proximity, at a distance modulus of 18.9 (Harries et al. 2003), makes high resolution studies feasible with $4-8 \mathrm{~m}$ telescopes and since the extinction is low, $E(B-V) \sim 0.07$, similar studies are also feasible in the important ultraviolet regime.

This combination of circumstances has led to a number of detailed studies of O-type stars in the SMC at optical and UV wavelengths (Walborn et al. 1995; Puls et al. 1996; Haser et al. 1998). Even more recently we have seen the publication of work related to a high resolution UV study of a sample of O-stars in the SMC (Walborn et al. 2000; Bouret et al. 2003; Hillier et al. 2003). In the present paper, and a companion paper (Evans et al. 2004; hereafter EVANS04), we extend this work into the domain of the B-type stars in the SMC. There are

Send offprint requests to: C. Trundle, e-mail: ct@ing.iac.es important reasons for this extension, among them the fact that the B-type stars are important contributors to the UV spectrum of continuous starforming and intermediate age (20-50 Myr) stellar populations (deMello et al. 2000). In fact the UV spectra of the stars discussed in the present paper have been used to constrain the age of the metal poor super star cluster NGC 1705-1 (Vazquez et al. 2003). However what is more relevant to the present paper is that B-type supergiants in the SMC provide important information on mass-loss and stellar evolution in a low metallicity environment.

The surface compositions of B-type supergiants are a key test of stellar evolution models since mixing and mass-loss processes may alter their surface $\mathrm{CNO}$ abundances, especially that of nitrogen. The SMC is an ideal laboratory in this respect since its pristine nitrogen abundance is about $1 / 30$ th to $1 / 50$ th of solar and enhancements are correspondingly easier to detect. Enhanced nitrogen abundances have already been observed in Galactic and Magellanic Cloud B-type supergiants indicating some mixing of CNO-cycled material to the stellar surface (Gies \& Lambert 1992; Lennon et al. 1991, 1996, 1997, 2003; Fitzpatrick \& Bohannan 1993; McErlean et al. 1999; Dufton et al. 2000). Moreover, Lyubimkov (1991) and later Gies \& Lambert (1992) found that Galactic main-sequence 
B-type stars also show such $\mathrm{N}$ enhancements in their spectra. Whether the mechanism which causes this mixing of core processed material to the photosphere is the presence of a blueloop evolutionary phase (Schaller et al. 1992) or due to stellar rotation (Denissenkov 1994; Talon 1997) is still uncertain. At present much effort is being put into the development of stellar evolution models which include rotationally induced mixing (Heger \& Langer 2000; Maeder \& Meynet 2000, 2001). Such models have had success in predicting the correct dependence of the blue to red supergiant $(\mathrm{B} / \mathrm{R})$ ratio on metallicity, previously a problem for models without rotation. This ratio is an important factor in stellar and galactic evolution models as it represents the relative lifetimes of hot and cool core helium burning phases. Eggenberger et al. (2002) showed that evolution models including rotationally induced mixing (Maeder \& Meynet 2000) seem to reproduce the observed B/R ratio at Galactic, LMC and SMC metallicities, as suggested by Langer \& Maeder (1995). Lennon et al. (2003; hereafter LEN03) investigated surface abundances of a sample of lower luminosity B-type giants in the SMC, and discussed these in the context of evolution with rotation. Whether these evolutionary models can reproduce the enhanced nitrogen abundances and rotational velocities observed in supergiants/giants in a low metallicity regime will be explored in this paper.

Blue supergiant stars, by virtue of their low bolometric correction and high intrinsic luminosity, are the most accessible stars to study at visible wavelengths. The first chemical compositions of individual stars in the Magellanic Clouds were determined from B- to F-type supergiants by Przybylski (1968, 1971), Wolf $(1972,1973)$ and Osmer (1972, 1973), but with mixed results. Osmer (1973) concluded, using simple curve of growth arguments, that since the metal lines in a B1.5 and B2.5 supergiant were approximately a factor of two lower than their Milky Way counterparts then their metallicities must be at least a factor of four below solar. This is remarkably close to the now commonly accepted value of one-fifth solar and its accuracy is probably due in part to the care with which galactic standards were chosen as comparison stars. Subsequently blue supergiants have provided important insights into the current chemical makeup of many galaxies in the Local Group and beyond (Monteverde et al. 1996, 1997, 1998, 2000; Venn et al. 1995, 1999, 2000, 2001, 2003a,b; McErlean et al. 1999; Dufton et al. 2000; Smartt et al. 2001; Bresolin et al. 2001, 2002a,b; Trundle et al. 2002; Urbaneja et al. 2003) using somewhat more sophisticated modeling techniques. Lennon et al. (1991) used plane-parallel non-LTE methods to analyse a B-type supergiant in each Cloud, this being followed by a more comprehensive non-LTE study of 48 B-type supergiants in the SMC by Dufton et al. (2000). Nevertheless as discussed by McErlean et al. (1998) the effect of microturbulence and the neglect of the stellar wind in this plane-parallel approach are significant sources of uncertainties in their results. With the recent development of stellar atmosphere codes which treat in a unified manner both the photosphere and wind of massive stars, it is now possible to include the effects of the stellar wind (Santolaya-Rey et al. 1997; Herrero et al. 2002; Repolust et al. 2003 - FASTWIND; Hillier \& Miller 1998 - CMFGEN; Pauldrach et al. 2001 wm-basic). We use FASTWIND in the present paper which in addition to determining the chemical composition of our sample, enables us to derive estimates for their mass-loss rates and wind momenta.

Kudritzki et al. (1995) introduced the Wind-Momentum Luminosity Relationship (WLR) as a method of determining distances out to the Virgo and Fornax Clusters through the spectral analysis of hot, massive stars. Line-driven wind theory implies that photon momentum transfer through metal line absorption is driving the stellar wind. As the WLR is based on line-driven wind theory, it follows that this relationship should be dependent on metallicity. Studies of O-type supergiants in the SMC and LMC have confirmed this metallicity dependence. Additionally, analyses of Galactic early-type supergiants have shown some dependence on spectral type (Puls et al. 1996; Kudritzki et al. 1999). More recent analyses of Bouret et al. (2003) and Repolust et al. (2003) show a distinct difference in the wind momenta of O-type supergiants to that of giants and dwarfs in both the Galaxy and Magellanic Clouds. In this paper we will compare the WLR of our sample with that of stars in the Galaxy and with the predictions of theory.

\section{Observations and data reduction}

High resolution echelle spectra were obtained for eight SMC B-type stars covering the spectral range of B0-B5, using the UVES spectrograph on the VLT. The data were taken during twilight on a 3 night run in November 2001, see the observational details in Table 1. UVES is a two arm crossdispersed echelle spectrograph, the red arm of which contains a mosaic of two chips. EEV CCD-44 $2 \mathrm{k} * 4 \mathrm{k}$ chips are used in both arms along with a MIT-LL CCID-20 CCD chip in the red arm which reduces the fringing beyond $7000 \AA$. The useful wavelength ranges obtained from the three chips were 3750-5000 $\AA, 5900$ $7700 \AA$ and $7750-9600 \AA$. These CCD chips have a pixel size of $15 \mu \mathrm{m}$ and a slit width of 1.5 arcsec which was used to give a resolving power of approximately 20000 . The spectra were later binned to a dispersion resolution of $0.2 \AA$ per pixel.

The 2-dimensional CCD frames were transformed to multiple order spectra using the UVES pipeline software. This was done in an interactive mode through the ECHELLE/UVES context of MIDAS. Th-Ar arc lamp exposures were used to define echelle order positions and wavelength calibrate the spectra. A constant bias level and median background were subtracted from the flat-fields which were then extracted in pixel-order space. The stellar spectra were reduced using both average and optimal extraction methods. In the blue region our analysis utilised the optimally extracted spectra, however due to the magnitude of the fringing in the red region the averaged extracted data proved to be more useful. The final merged spectra and individual orders were then processed using an automated IDL routine to filter out the cosmic rays.

Normalisations, corrections for radial velocity shifts and equivalent width $(E W)$ measurements were carried out in DIPSO, the spectrum analysis procedure of STARLINK. The measured equivalent widths for the lines used in the abundance analysis are given in Table 2. Although the REDUCE/UVES routine normalised and merged the echelle orders, these merged spectra were checked manually. Low order polynomials were 
Table 1. Observational details. Identification numbers are from Azzopardi \& Vigneau (1982; AV\#) and Sanduleak (1968; Sk\#). Spectral types adopted from Lennon (1997). Absolute Magnitudes $\left(M_{v}\right)$ are calculated using $V$ and $(B-V)$ magnitudes from Garmany et al. (1987; $\left.{ }^{1}\right)$ and Massey $\left(2002 ;^{2}\right)$ and $(B-V)_{0}$ values from Fitzpatrick \& Garmany (1990). The adopted distance modulus is 18.9 (Harries et al. 2003$)$. The average $S / N$ ratios for the VLT/UVES data are given, but the actual $S / N$ varied across the orders. Heliocentric corrected radial velocities are presented for both the VLT/UVES and NTT/EMMI datasets. Note there is no significant variation in radial velocities from the two datasets and thus no obvious indication of binary stars amongst the sample. In addition we note that the quoted $v \sin i$ values are upper limits and are dominated by macroturbulence as discussed in Sect. 2.

\begin{tabular}{lllllcccccc}
\hline \hline STAR & Alternative & Spectral & $V$ & $B-V$ & $M_{v}$ & \multicolumn{2}{c}{$S / N$} & \multicolumn{2}{c}{$v_{\text {lsr }}$} & $v \sin i$ \\
& ID & Type & & & & $B$ & $R$ & UVES & EMMI & $\left(\mathrm{km} \mathrm{s}^{-1}\right)$ \\
\hline AV 215 & Sk 76 & BN0 Ia & $12.69^{2}$ & -0.09 & -6.61 & 120 & 110 & $154 \pm 10$ & $159 \pm 15$ & 91 \\
AV 104 & & B0.5 Ia & $13.17^{2}$ & -0.16 & -5.82 & 150 & 120 & $163 \pm 12$ & $165 \pm 28$ & 80 \\
AV 216 & & B1 III & $14.22^{2}$ & -0.13 & -5.08 & 120 & 100 & $203 \pm 14$ & & 77 \\
& Sk 191 & B1.5 Ia & $11.86^{1}$ & -0.04 & -7.41 & 170 & 140 & $130 \pm 24$ & $134 \pm 17$ & 96 \\
AV 210 & Sk 73 & B1.5 Ia & $12.60^{2}$ & -0.02 & -6.73 & 180 & 140 & $173 \pm 8$ & $192 \pm 12$ & 65 \\
AV 18 & Sk 13 & B2 Ia & $12.46^{1}$ & 0.03 & -7.00 & 170 & 120 & $148 \pm 10$ & $127 \pm 13$ & 49 \\
AV 362 & Sk 114 & B3 Ia & $11.36^{1}$ & -0.03 & -7.82 & 190 & 100 & $208 \pm 14$ & $214 \pm 10$ & 51 \\
AV 22 & Sk 15 & B5 Ia & $12.25^{1}$ & -0.10 & -6.62 & 140 & 120 & $139 \pm 12$ & $109 \pm 16$ & 46 \\
\hline
\end{tabular}

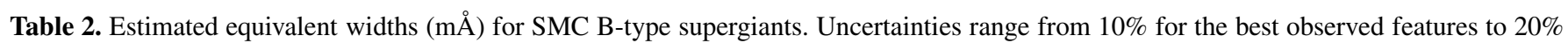
for the weaker features.

\begin{tabular}{lcccccccc}
\hline \hline Line & AV 215 & AV 104 & AV 216 & Sk 191 & AV 210 & AV 18 & AV 362 & AV 22 \\
\hline C II 4267 & 25 & $52:$ & 49 & 48 & 83 & 81 & 53 & 55 \\
C II 6578 & & & & & 105 & 154 & 120 & 72 \\
C II 6580 & & & & & 116 & 119 & 70 & 77 \\
N II 3995 & 68 & 49 & 40 & 137 & 173 & 169 & 95 & 81 \\
N II 4447 & & & & 47 & 69 & 47 & 39 & 26 \\
N II 4601 & & & & 50 & 59 & 49 & & \\
N II 4607 & & & & 29 & 61 & 46 & & \\
N II 4614 & & & & & 42 & 22 & & \\
N II 4621 & 31 & & & 33 & 51 & 25 & & \\
N II 4630 & 73 & 48 & & 95 & 158 & 103 & 53 & 47 \\
O II 4072 & 57 & 88 & 87 & 96 & 86 & 69 & & \\
O II 4076 & 44 & 121 & 87 & 141 & 106 & 88 & & \\
O II 4317 & & 40 & & 95 & 72 & 61 & & \\
O II 4320 & & 36 & & 87 & 87 & 63 & & \\
O II 4367 & 38 & 49 & & 92 & 88 & 61 & & \\
O II 4415 & & 121 & 86 & 166 & 132 & 109 & & \\
O II 4417 & & 31 & 70 & 71 & 81 & 66 & & \\
O II 4591 & 54 & 67 & 29 & 86 & 58 & 40 & & \\
O II 4596 & 58 & 50 & 43 & 62 & 43 & 33 & & \\
O II 4638 & & 40 & & 141 & 81 & 169 & & \\
O II 4641 & & 137 & & 250 & 92 & 93 & & \\
O II 4662 & 55 & 69 & 50 & 151 & 207 & 77 & & \\
Mg II 4481 & $80:$ & 65 & 46 & 75 & 114 & 93 & 182 & 217 \\
Si II 4128 & & & & & & $30:$ & 73 & 94 \\
Si II 4131 & & & & & & $70:$ & 79 & 96 \\
Si III 4553 & 168 & 161 & 94 & 266 & 264 & 199 & 56 & 58 \\
Si III 4568 & 129 & 120 & 78 & 206 & 211 & 174 & 47 & 45 \\
Si III 4575 & 64 & 54 & 40 & 108 & 113 & 95 & 21 & \\
Si IV 4116 & 212 & 111 & 60 & 47 & 34 & & & \\
\hline
\end{tabular}

fitted to the continuum of each order for normalisation, the orders were then merged using appropriate weightings. In most cases, the automatically and manually reduced data were in good agreement. Nevertheless in measuring the equivalent widths of lines in the overlapping regions of different orders and in analysing the Balmer lines, more weight was given to the manually reduced spectra.

The metal and diffuse helium absorption lines were fit using a non-linear least squares technique with a Gaussian 
profile. For isolated, well observed lines the full-widthhalf-maximum (FWHM) and position were allowed to vary. Blended lines such as the C II $4267 \AA$ and Mg II $4481 \AA$ were treated as multiple Gaussians with fixed wavelength separations and adopting the mean FWHMs. For well observed lines error estimates of $10 \%$ are appropriate, however blended and weak lines have errors of $20 \%$ or more. The signal-to-noise ratio $(S / N)$ obtained was measured throughout the spectral range of each arm in regions of continuum with no observed spectral lines. In the blue region $S / N$ is typically 150 or higher and in the red all spectra have a $S / N$ greater than 100 . Radial velocity shifts were measured from the positions of the well observed lines and are presented in Table 1 along with the mean $S / N$ ratios.

Additionally, medium resolution spectra of the seven supergiants were previously obtained using the NTT telescope in remote observing mode with the ESO Multi Mode Instrument (EMMI). The reduction methods of this dataset were discussed in Lennon (1997) and all equivalent widths were remeasured here using the technique described above. Each object was observed for between 10-15 min. Depending on its visual magnitude and the weather conditions at the time, these exposure times gave signal-to-noise ratios greater than 60 in the red and 90 in the blue. The equivalent widths measured from this dataset agree with the UVES dataset to within 5-20\% depending on the quality of the spectra. Comparison of radial velocity measurements from the EMMI and UVES datasets show no signs of binaries amongst the stellar selection (see Table 1). However from STIS/HST target acquisition images, EVANS04 noticed a companion to AV 216, the faintness of which suggests that it is a main-sequence star. Indeed some asymmetry was observed in the $\mathrm{H}_{\alpha}$ and He I $4471 \AA$ profiles (see Figs. 3 and 2). The additional dataset was mainly used for a check on our UVES data. However due to some nebular contamination of the $\mathrm{H}_{\alpha}$ profiles of AV 362 and AV 22, the EMMI dataset was used to derive the mass-loss rate of these two stars. There was also some nebular contribution observed in the $\mathrm{H}_{\alpha}$ profile of Sk 191, and we have taken this into account in fitting the data.

The projected rotational velocity for each $\operatorname{star}, v \sin i$, was determined from a selection of seven unblended metal lines by fitting theoretical profiles to the observed spectra. The theoretical profiles were convolved with a Gaussian profile to account for the instrumental broadening. The profile was then convolved with a rotational line profile function, this has been described in detail by Rucinski (1990) and more recently by Gray (1992). Howarth et al. (1997) and Ryans et al. (2002) found that the broadening of spectral features in B-type supergiants was dominated by macroturbulence. Indeed our projected rotational velocity estimates listed in Table 1 show a similar behaviour. Ryans et al. (2002) also estimated that the typical projected rotational velocity was of the order $10-20 \mathrm{~km} \mathrm{~s}^{-1}$. Hence the values for $v \sin i$ tabulated in Table 1 should be considered as indicative of the width of the spectral features and as upper limits on the projected rotational velocities.

\section{Model atmospheres}

"Unified model atmosphere" codes such as those mentioned in the introduction have an advantage over plane-parallel codes (viz. TLUSTY - Hubeny \& Lanz 1995). This is due to treating the atmosphere in a consistent manner by including a smooth transition, from a pseudo-hydrostatic photosphere to an outer expanding atmosphere - "the wind". We have opted to use FASTWIND (Fast Analysis of STellar atmospheres with WINDs), a spherically symmetric non-LTE code first introduced by Santolaya-Rey et al. (1997). FASTWIND has been developed to include an approximated treatment of metal line blocking and -blanketing (Herrero et al. 2002; Repolust et al. 2003).

The main advantage of FASTWIND over more sophisticated codes such as CMFGEN (Hillier \& Miller 1998) is the short computational time it takes to calculate models for a significant parameter space. Fast computational time allows for quantitative spectroscopic analyses of large samples of massive stars whilst still accounting for the stellar wind. This is achieved by parameterising the temperature structure using the non-LTE Hopf function described by Santolaya-Rey et al. (1997) which includes the effects of sphericity and mass-loss:

$q_{\mathrm{N}}^{\prime}\left(\tau_{\mathrm{R}}^{\prime}\right)=\frac{\tau_{\mathrm{R}}^{\prime}}{\tau_{\mathrm{R}}} q_{\mathrm{N}}\left(\tau_{\mathrm{R}}\right)$

where $\tau_{\mathrm{R}}^{\prime}$ and $q_{\mathrm{N}}^{\prime}$ are the spherical generalisation of the optical depth, $\tau_{\mathrm{R}}$, and the plane parallel Hopf function, $q_{\mathrm{N}}\left(\tau_{\mathrm{R}}\right) . q_{\mathrm{N}}\left(\tau_{\mathrm{R}}\right)$ and $\tau_{\mathrm{R}}^{\prime}$ are defined by

$q_{\mathrm{N}}\left(\tau_{\mathrm{R}}\right) \approx q_{\infty}+\left(q_{0}-q_{\infty}\right) \exp \left(-\gamma \tau_{\mathrm{R}}\right)$

and

$\mathrm{d} \tau_{\mathrm{R}}^{\prime}=\chi_{\mathrm{R}}(r)\left(\frac{R_{\star}}{r}\right)^{2} \mathrm{~d} r$

where $q_{\infty}, q_{0}$ and $\gamma$ are fit parameters to the run of the Hopf function, $q_{\mathrm{N}}$, as a function of Rosseland opacity, $\left(\chi_{\mathrm{R}}\right)$. For the models discussed in this paper we have adopted Hopf parameters from the parameterisation of non-LTE line-blanketed (including Iron) TLUSTY models calculated at Queen's University Belfast (Ryans, private communication). The TLUSTY grid covered a temperature range of $15 \mathrm{kK}$ to $33 \mathrm{kK}$ and logarithmic surface gravities from 4.5 dex down to the Eddington limit. The abundances in this grid were baseline SMC abundances (viz. $[\mathrm{Fe} / \mathrm{H}]=6.80 \mathrm{dex}$ ). The flux conservation reached by using the TLUSTY temperature stratification was typically of the order of 1-2\%, however for stars with high mass-loss rates this was higher at $\sim 3 \%$.

FASTWIND has a similar philosophy to that of the line formation code DETAIL (Butler \& Giddings 1985), i.e. the code is data driven. The calculated models for this work include atomic data for hydrogen, helium, carbon, nitrogen, oxygen, magnesium and silicon. The atomic models and Stark broadening data implemented in the line formation of hydrogen and helium are described in full in Santolaya-Rey et al. (1997). In the case of silicon, nitrogen, and oxygen, the metal ion populations and line profiles were calculated using the atomic data of Becker \& Butler $(1988,1989,1990)$. The atomic data used for C II are from Eber \& Butler (1988), and the Mg II is from Mihalas (1972). In most cases these datasets needed some modification to ensure convergence for the temperatures and gravities considered here, however tests showed that these had negligible impact on the resultant line strengths. 
For Si II we initially used the silicon model atom as described by Becker \& Butler (1990). This model essentially uses the atomic levels adopted by Kamp (1978), but with updated atomic data for bound-bound and bound-free transitions (see also Lennon et al. 1986). However comparisons of TLUSTY and FASTWIND calculations in the low mass-loss rate limit (see below) showed severe discrepancies for the Si II equivalent widths. This was traced to the simplified atomic model for this ion in the FASTWIND calculations. We therefore replaced our 12-level Si II model ion with a more complete 34-level model ion. This has important consequences for the determination of the effective temperatures of B-type stars, in that for the mid to late B-type stars (B2-B9) the derived temperatures will be cooler using the new Si II model atom. However it is beyond the scope of this paper to include a detailed discussion of this and so it will be reported in future work.

To check the validity of our results from FASTWIND, we have carried out preliminary tests with the plane-parallel code TLUSTY and unified model atmosphere code, CMFGEN (Hillier $\&$ Miller 1998). Choosing a star with a thin-wind, AV 104, and a thick-wind, AV 215, we analysed these stars with TLUSTY and CMFGEN, respectively. No major discrepancies were found in these analysis with any differences being within the estimated errors. This is reassuring given the different atomic data and more importantly the various physical assumptions each code adopts (viz. degree of line-blanketing, treatment of wind, calculation of formal solution etc.).

Finally it should be noted that some of the projected rotational velocities listed in Table 1 are relatively large. Therefore, an approach allowing for the variation of atmospheric structure over the stellar surface (as used for example by Howarth \& Smith 2001) might be warranted. However as discussed above, the actual rotational velocities are likely to be considerably smaller and the assumption of spatial homogeneity should be appropriate.

\section{Photospheric and wind analysis}

\subsection{Stellar parameters}

The atmospheric and wind parameters of our stellar sample are presented in Tables 3 and 4, respectively. The methods for determining these parameters are described in the following section; however one should note that they are not derived independently but through an iterative process.

In order to allow an initial assessment of the parameter space appropriate for each of the SMC stars, a coarse grid of FASTWIND models were calculated to cover the spectral range B0-B5. For each spectral bin, we adopted one effective temperature and a range of surface gravities, spanning $0.5 \mathrm{dex}$. The microturbulence adopted was $10 \mathrm{~km} \mathrm{~s}^{-1}$ as appropriate for B-type supergiants, if derived from the silicon spectrum (see Sect. 4.1.3). The stellar radius was chosen based on the effective temperature of the model as well as bolometric corrections and colors taken from Fitzpatrick \& Garmany (1990). The $\beta$-parameter was fixed at 1 and terminal velocities were taken from Kudritzki \& Puls (2000), where a calibration of terminal velocities with spectral type for Galactic stars is presented.
Five mass-loss rates for each spectral bin were adopted, covering thin to thick winds. To obtain initial estimates of the parameters for each star, the appropriate set of models for its spectral type was chosen and a comparison with the observed data gave an approximate mass-loss rate and surface gravity. Once initial values were defined, finer, smaller grids were run which were appropriate to each individual star.

\subsubsection{Effective temperature, $T_{\text {eff }}$}

In early B-type stars, the silicon lines are the spectral features most sensitive to changes in effective temperature, $T_{\text {eff }}$ (see Figs. 1 and 2 for the variation of the silicon ions line strength with spectral type). The standard method of determining $T_{\text {eff }}$ in B-type stars is to search for an ionisation balance between consecutive stages of silicon ionisation (Kilian et al. 1991). Although these lines are sensitive to the silicon abundance, by considering the ratio of the $E W$ 's for neighbouring ionisation stages we eliminate, to first order, this dependence on abundance. Initially $T_{\text {eff }}$ was constrained by fitting theoretical profiles to the Si lines, this was then followed by a comparison between $E W$ 's of theoretical and observed profiles. The error in this method due to observational constraints and random errors is typically $\pm 1.5 \mathrm{kK}$, with a few exceptions as discussed below.

For the hotter stars of our sample (i.e. B0-B1.5) the prominent ionisation stages of silicon are Si III and IV. In the case of AV 104 and AV 215, the He II lines at 4200 and $4542 \AA$ were used as further constraints on the temperature, assuming a normal helium abundance. Given this additional check on $T_{\text {eff }}$ the error in these measurements is $\pm 1.0 \mathrm{kK}$. In AV 362 and AV 22, the coolest objects in our sample, Si II and III are the dominant silicon ions with Si IV absent from the spectra. Unfortunately neither the Si II nor Si IV features are very strong in B2-type stars at low metallicities such as that of the SMC, in fact we only observe noise features in the region of these lines in AV 18. Although we could not accurately fix an ionisation balance, the absence of Si II and IV features provided lower and upper limits, respectively, for the temperature of AV 18. Thus, the error we adopt in our temperature estimate for AV 18 is slightly larger than for the rest of our sample $( \pm 2.0 \mathrm{kK})$.

The inclusion of sphericity and line-blanketing in the analysis of O-type dwarfs and supergiants has introduced a new temperature scale for O-type stars (Martins et al. 2002; Herrero et al. 2002; Crowther et al. 2002; Repolust et al. 2003). Compared to plane-parallel, unblanketed models the temperatures derived for O-type stars are up to $8.0 \mathrm{kK}$ cooler, when line-blanketed, spherical models are applied. Herrero et al. (2002) noted that this discrepancy decreased to $2.0 \mathrm{kK}$ at the O9.5 Ia spectral type. For the B0-B2 -type stars in our sample no notable difference was observed in our temperature estimates when compared to the calibration of Dufton et al. (2000) for B-type supergiants (this was also seen in Repolust et al. 2003). However for the B3 and B5 star, our temperature estimates were much lower than their temperature scale. This is probably a result of our improved Si II model, which for a given temperature, weakened the Si II line strength. Additionally 

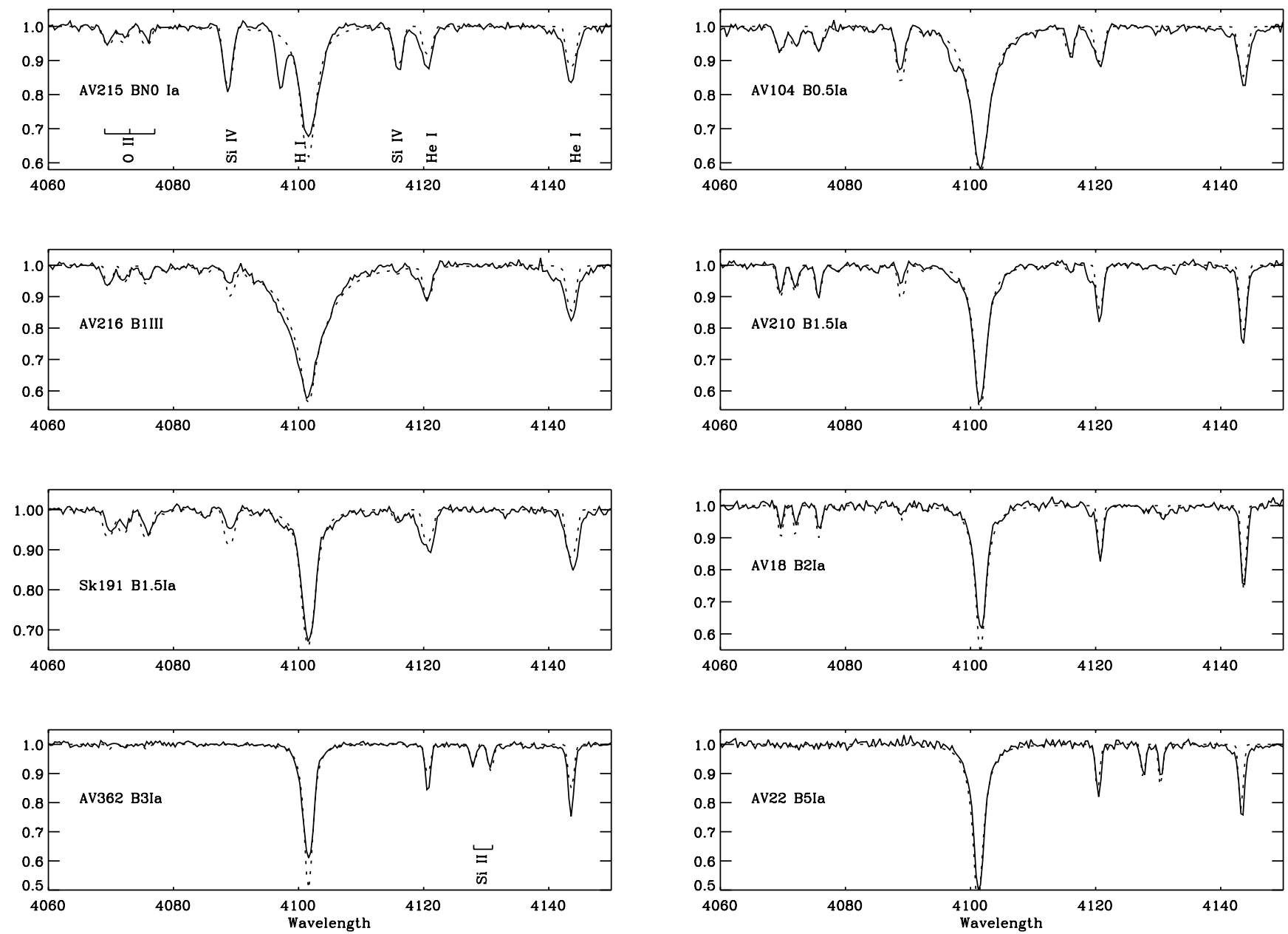

Fig. 1. Profile fits to spectral region 4060-4150 Å. Observed and theoretical data are shown as solid (-) and dotted ( $\cdots)$ lines, respectively. Note the fits to the wings of the $\mathrm{H}_{\delta}$ line which were used to fix $\log \mathrm{g}$ and the varying strengths of the Si II and IV lines that were the temperature indicators. Also note the absence of the O II multiplet at $4070 \AA$ in the coolest stars AV 362 and AV 22. O II is not observed in the spectra of such cool stars, preventing the determination of $\xi_{0}$.

Table 3. Derived atmospheric parameters for SMC B-type supergiants. The microturbulence from both the Si III and O II lines are presented, however the silicon microturbulence was adopted in our analysis. A microturbulence of $10 \mathrm{~km} \mathrm{~s}^{-1}$ was adopted for AV 22, due to the difficulties in constraining $\xi$ from the metal lines in its' spectrum. $M_{\text {evol }}$ are estimated from the stellar evolution tracks of Maeder \& Meynet (2001), see discussion in Sect. 6.2. The errors quoted are the typical random errors as discussed in Sect. 4.

\begin{tabular}{|c|c|c|c|c|c|c|c|c|c|}
\hline Star & $\begin{array}{l}\text { Spectral } \\
\text { Type }\end{array}$ & $\begin{array}{c}T_{\text {eff }} \\
(\mathrm{kK})\end{array}$ & $\begin{array}{l}\log g \\
(\operatorname{cgs})\end{array}$ & $\begin{array}{c}R_{\star} \\
\left(R_{\odot}\right)\end{array}$ & $\begin{array}{l}M_{\text {spec }} \\
\left(M_{\odot}\right)\end{array}$ & $\begin{array}{l}M_{\mathrm{evol}} \\
\left(M_{\odot}\right)\end{array}$ & $\log \left(\frac{L_{\star}}{L_{\odot}}\right)$ & $\begin{array}{l}\xi_{\mathrm{Si}} \\
(\mathrm{km}\end{array}$ & $\begin{array}{l}\xi_{\mathrm{O}} \\
-1)\end{array}$ \\
\hline AV 215 & BN0 Ia & $27.0 \pm 1.0^{1}$ & $2.90 \pm 0.10$ & 30 & 26 & 39 & 5.63 & 12 & 17 \\
\hline AV 104 & B0.5 Ia & $27.5 \pm 1.0^{1}$ & $3.10 \pm 0.10$ & 20 & 19 & 26 & 5.31 & 11 & 20 \\
\hline AV 216 & B1 III & $26.0 \pm 1.5^{2}$ & $3.60 \pm 0.20$ & 16 & 36 & 20 & 5.00 & 0 & 15 \\
\hline Sk 191 & B1.5 Ia & $22.5 \pm 1.5^{2}$ & $2.55 \pm 0.15$ & 51 & 33 & 41 & 5.77 & 13 & 20 \\
\hline AV 210 & B1.5 Ia & $20.5 \pm 1.5^{2}$ & $2.40 \pm 0.15$ & 40 & 15 & 27 & 5.41 & 12 & 18 \\
\hline AV 18 & B2 Ia & $19.0 \pm 2.0^{3}$ & $2.30 \pm 0.20$ & 49 & 17 & 28 & 5.44 & 9 & 16 \\
\hline AV 362 & B3 Ia & $14.0 \pm 1.5^{4}$ & $1.70 \pm 0.15$ & 96 & 17 & 30 & 5.50 & 10 & \\
\hline AV 22 & B5 Ia & $14.5 \pm 1.5^{4}$ & $1.90 \pm 0.15$ & 53 & 8 & 19 & 5.04 & $10^{*}$ & \\
\hline
\end{tabular}

$T_{\text {eff }}$ determined from : ${ }^{1} \mathrm{Si}$ III/Si IV ionisation balance and He II fits;

${ }^{2} \mathrm{Si} \mathrm{III/Si} \mathrm{IV} \mathrm{ionisation} \mathrm{balance;}{ }^{3}$ Fits to Si III lines $;{ }^{4} \mathrm{Si}$ II $/ \mathrm{Si}$ III ionisation balance. 

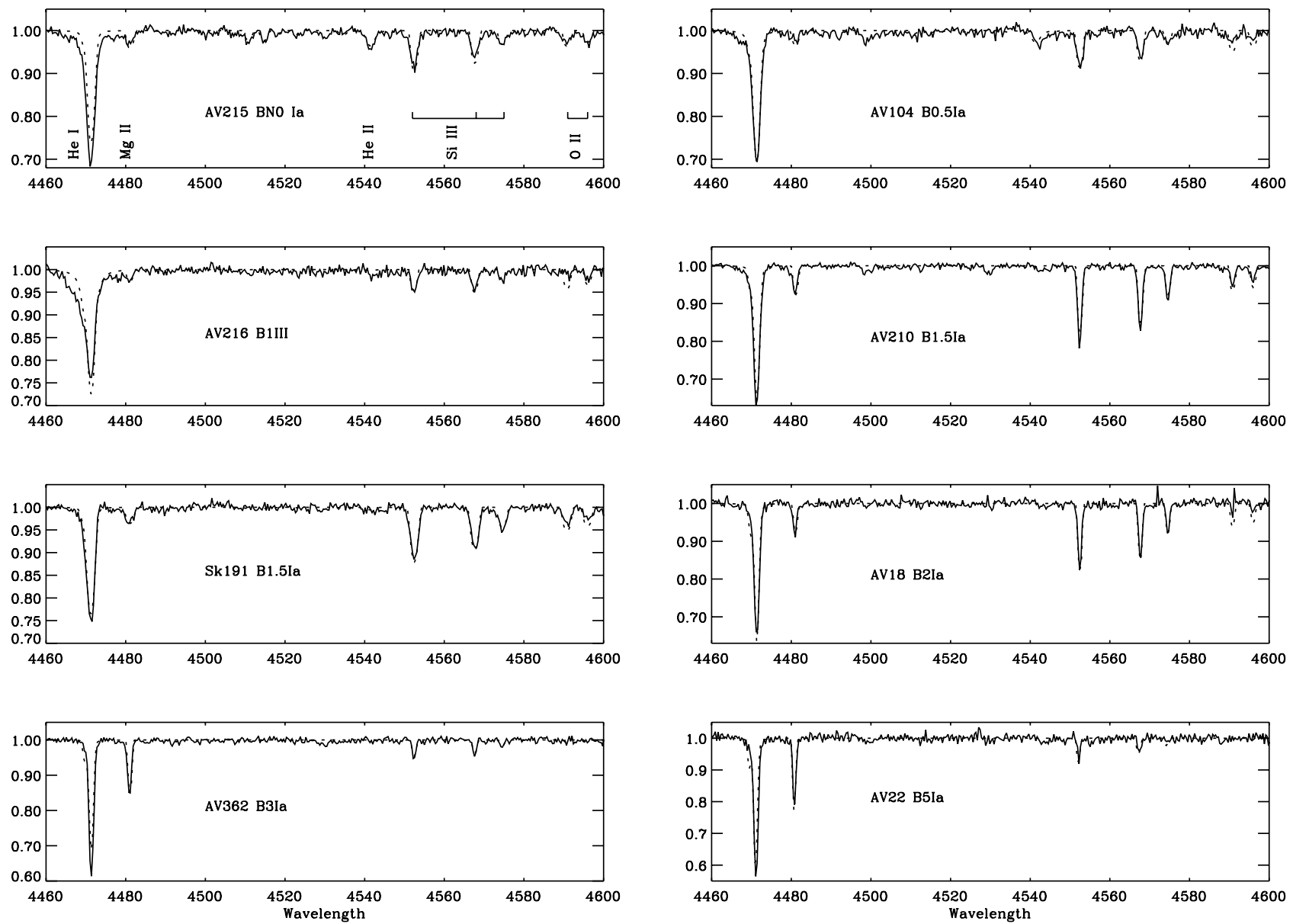

Fig. 2. Profile fits to spectral region 4460-4600 Å. Observed and theoretical data are shown as solid (-) and dotted ( . -) lines, respectively. Note the varying strengths of the Si III lines, which were used as temperature indicators. Also note the presence of the He II line at $4542 \AA$ in AV 215 and AV 104, which was used as an additional temperature diagnostic. The asymmetry in the He I 4471 line of AV 216 suggests some multiplicity.

Table 4. Derived wind parameters for SMC B-type supergiants. Escape velocities are taken from EVANS04 for AV 215, Sk 191 , AV 210 and AV 18. For the other four they are calculated in this work using the procedure outlined in EVANS04. Terminal velocities, $\left(v_{\infty}\right)$, for AV 215 , Sk 191, AV 210, and AV 18 are taken from EVANS04, as derived from the SEI method. For the rest of the sample, $v_{\infty}$ is calculated by adopting a $v_{\infty} / v_{\text {esc }}$ ratio of 2.65 for stars with $T_{\text {eff }} \geq 21.0 \mathrm{kK}$ and 1.40 for $T_{\text {eff }}<21.0 \mathrm{kK}$. The errors quoted are the typical random errors as discussed in Sect. 4 .

\begin{tabular}{llccccc}
\hline \hline Star & $\begin{array}{l}\text { Spectral } \\
\text { Type }\end{array}$ & $\begin{array}{c}\dot{M} \\
\left(10^{-6} M_{\odot} \mathrm{yr}^{-1}\right)\end{array}$ & $\begin{array}{c}v_{\infty} \\
\left(\mathrm{km} \mathrm{s}^{-1}\right)\end{array}$ & $\begin{array}{c}v_{\text {esc }} \\
\left(\mathrm{km} \mathrm{s}^{-1}\right)\end{array}$ & $\beta$ & $\begin{array}{c}\log \left(D_{\text {mom }}\right) \\
(\mathrm{cgs})\end{array}$ \\
\hline AV 215 & BN0 Ia & $1.35 \pm 0.14$ & $1400 \pm 100^{1}$ & $447^{1}$ & $1.4 \pm 0.2$ & $28.81 \pm 0.10$ \\
AV 104 & B0.5 Ia & $0.40 \pm 0.05$ & $1340 \pm 270^{2}$ & $506^{2}$ & $1.0 \pm 0.3$ & $28.18 \pm 0.15$ \\
AV 216 & B1 III & $0.12 \pm 0.02$ & $2410 \pm 480^{2}$ & $908^{2}$ & $1.4 \pm 0.3$ & $27.86 \pm 0.15$ \\
Sk 191 & B1.5 Ia & $0.68 \pm 0.07$ & $425 \pm 50^{1}$ & $374^{1}$ & $2.0 \pm 0.2$ & $28.11 \pm 0.10$ \\
AV 210 & B1.5 Ia & $0.20 \pm 0.05$ & $750 \pm 100^{1}$ & $290^{1}$ & $3.0 \pm 0.3$ & $27.52 \pm 0.15$ \\
AV 18 & B2 Ia & $0.23 \pm 0.03$ & $325 \pm 50^{1}$ & $284^{1}$ & $3.0 \pm 0.2$ & $27.66 \pm 0.10$ \\
AV 362 & B3 Ia & $0.80 \pm 0.08$ & $270 \pm 50^{2}$ & $192^{2}$ & $1.0 \pm 0.3$ & $28.12 \pm 0.15$ \\
AV 22 & B5 Ia & $0.23 \pm 0.02$ & $280 \pm 60^{2}$ & $198^{2}$ & $1.0 \pm 0.3$ & $27.47 \pm 0.15$ \\
\hline
\end{tabular}

Terminal and escape velocities as measured in ${ }^{1}$ EVANS04 and estimated in ${ }^{2}$ this work.

these two cooler stars have morphologically different spectra from which their spectral types have been derived, however they have very similar temperatures. The fact that the temperatures of these stars are indistinguishable between the two spectral types, is partly due to the lower luminosity and hence higher gravity of AV 22; this temperature "degeneracy" is also observed between AV 215 and AV 104 (B0 and B0.5 stars). An additional factor that may be coming into play here is 

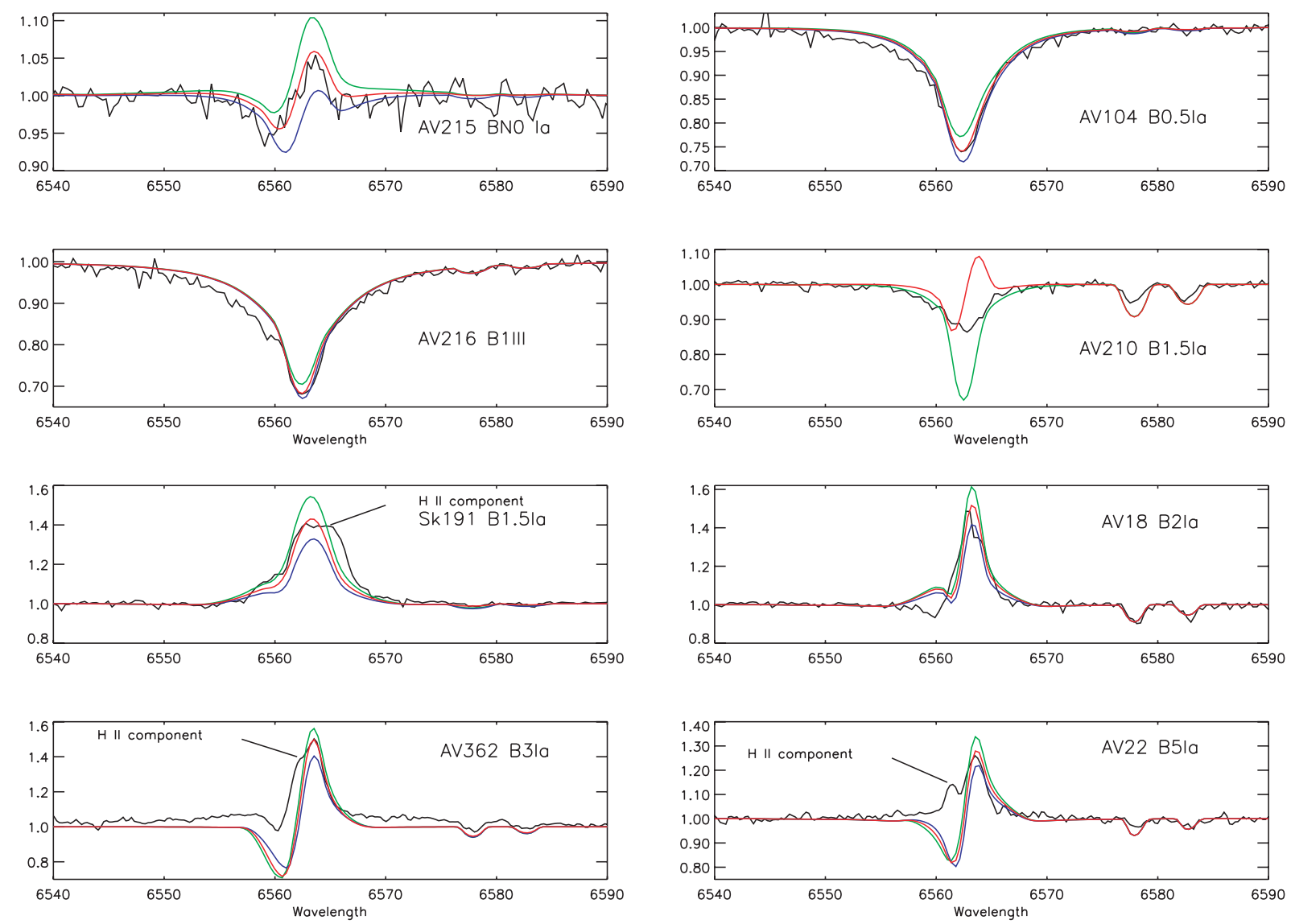

Fig. 3. $\mathrm{H}_{\alpha}$ profile fits for the target stars. Shown are the best fits (red line) using the parameters presented in Tables 3 and 4 . The green and blue lines represent a change in mass-loss rate of $\pm 15 \%$. However, for AV 210, the best fit adopting a $\beta$-parameter of 3 (red line) and 1 (green line) are plotted. Note the asymmetry in the $\mathrm{H}_{\alpha}$ profile of AV 216, this suggests that the companion star is contaminating AV 216 's spectrum. For AV 18, Sk 191, AV 362 and AV 22 the best fit was chosen by fitting the emission peak. The latter three have nebular contributions in the $\mathrm{H}_{\alpha}$ profile which were avoided in the fitting procedure. Note how for AV 362 and AV 22 the models have a large absorption trough which is not present in the observed spectrum.

that AV 215 and AV 362 both have very high mass-loss rates. Herrero et al. (2002) and Evans et al. (2003) observed that for extreme supergiants the effective temperature derived is much lower than for normal supergiants. Voels et al. (1989) also observed a reduction of $2.0 \mathrm{kK}$ in the temperature of $\alpha$ Cam, an O9.5 Ia type star, with the inclusion of the stellar wind in the analysis. They attributed this to "wind-blanketing", the backscattering of radiation onto the photosphere from the stellar wind (see also Repolust et al. 2003).

\subsubsection{Surface gravity, $\log g$}

The logarithmic surface gravity, $\log g$, is determined in an iterative process with $T_{\text {eff. }}$. The sensitivity of the Balmer line wings to gravity provide an excellent tool for deriving $\log g$. As $\mathrm{H}_{\alpha}$ and $\mathrm{H}_{\beta}$ are formed higher up in the atmosphere than $\mathrm{H}_{\gamma}$ and $\mathrm{H}_{\delta}$, these lines are more affected by the wind. Therefore the latter lines were used to determine the surface gravity with the fitting uncertainty being typically \pm 0.05 dex. However the uncertainty in the effective temperature estimates induces an additional though correlated uncertainty in $\log g$. These error estimates are presented in Table 3 along with the logarithmic surface gravities, while the theoretical and observed
$\mathrm{H}_{\delta}$ profiles are shown in Fig. 1. These measured $\log g$ values are in fact only the effective gravity, which is reduced due to the centrifugal acceleration by $(v \sin i)^{2} / R_{\star}$. The correction to the derived surface gravities due to the centrifugal force is less than 0.03 dex for these stars as a result of the low projected rotational velocities and large stellar radii. As discussed in Sect. 2, the measured $v \sin i$ value is an upper limit to the projected rotational velocity and so the correction to the surface gravity for these B-type supergiants is negligible. In addition, we note that the gravity for AV 216 may be overestimated due to the companion star, which is possibly a main-sequence star (see Sect. 2) and would therefore have broader Balmer wings.

\subsubsection{Microturbulence, $\xi$}

Microturbulence, $\xi$, is introduced to ensure no variation of abundance with line strength from a particular ionic species. In early B-type stars there is a sufficient number of $\mathrm{O}$ II and $\mathrm{Si}$ III lines covering a range of line strengths from which to determine microturbulence. However, the estimates from different metal species can differ by up to $10 \mathrm{~km} \mathrm{~s}^{-1}$ (see Vrancken et al. 1998, 2000; McErlean et al. 1999; Trundle et al. 2002). This effect was again observed in our sample where the 
microturbulence deduced from the $\mathrm{O}$ II lines, $\xi_{\mathrm{O}}$, were on average $7 \mathrm{~km} \mathrm{~s}^{-1}$ higher than those derived from the Si III lines, $\xi_{\mathrm{Si}}$. Whilst the oxygen lines have a significant range of $E W$ 's from which to determine the microturbulence, this $E W$ range is not present in one particular multiplet but over a number of multiplets. Therefore the scatter in oxygen abundances from one line to another is not simply due to microturbulence but to uncertainties in the modelling (e.g. errors in the oscillator strengths or departure coefficients between different multiplets). This is not the case for the Si III multiplet which has three lines at close wavelength intervals due to the small difference in the energy levels involved in these transitions, as such they are effected by the same radiation field. Therefore any errors in the oscillator strengths or departure coefficients would be similar for each line. The microturbulent velocities from both elements are presented in Table 3 , although we adopt the microturbulence derived from the Si III triplet at $4560 \AA$ (4553, 4568, $4575 \AA)$. Due to the low $T_{\text {eff }}$ of AV 362 and AV 22 no O II lines were observed and so $\xi_{\mathrm{O}}$ could not be derived (see Fig. 1). In addition, for AV 22 we had difficulty constraining the silicon microturbulence as the Si III $4575 \AA$ line was not visible in the spectrum and thus there was not a significant enough range in line strength to determine $\xi_{\mathrm{Si}}$. Therefore, we adopted a microturbulence of $10 \mathrm{~km} \mathrm{~s}^{-1}$. A discussion of the effect of microturbulence on the abundances of our supergiant sample is given in Sect. 5.

In previous non-LTE analyses of supergiants the microturbulences determined have been significantly lower than values derived from LTE analyses, which tended to be above the speed of sound (Becker \& Butler 1989; Gies \& Lambert 1992; McErlean et al. 1999; Vrancken et al. 2000; Trundle et al. 2002). Although for main-sequence B-type stars the value of $\xi$ is typically lower than $5 \mathrm{~km} \mathrm{~s}^{-1}$, in supergiants the microturbulence is still quite high even with the inclusion of non-LTE effects. It was suggested by Lennon et al. (1991) and investigated by Kudritzki et al. (1992) that the microturbulence may be reduced for supergiants by the consideration of the wind outflow in these stars. However, it is evident from the values in Table 3 that microturbulence is still needed in analyses which include the non-LTE effects and winds of supergiants. In fact the inclusion of spherical symmetry and treatment of the stellar wind in our model atmospheres doesn't appear to have any effect on the magnitude of microturbulence. From a plane-parallel non-LTE analysis of a group of galactic B-type supergiants, McErlean et al. (1999) found an average microturbulence of $11 \mathrm{~km} \mathrm{~s}^{-1}$ and this is consistent with our values. For AV 216, the giant in our sample, the Si III lines were compatible with $\xi=0 \mathrm{~km} \mathrm{~s}^{-1}$, while the $\mathrm{O}$ II lines implied a $\xi=15 \mathrm{~km} \mathrm{~s}^{-1}$. We note that adopting the latter value would imply a higher effective temperature estimate for the Si III/IV ionisation equilibrium. In turn this would lead to He II line strengths incompatible with the observed helium spectrum of AV 216.

\subsubsection{Radius, luminosity, and mass}

Apparent $V$-magnitudes were taken, where possible, from the UBVR CCD survey of Massey (2002). For the brighter targets which were not covered in this survey, $m_{v}$ was taken from Garmany et al. (1987). Absolute magnitudes were than deduced using $(B-V)_{0}$ from the calibration of Fitzpatrick \& Garmany (1990) and adopting an SMC distance modulus (DM) of 18.9 (Harries et al. 2003). As the intrinsic colours of Fitzpatrick \& Garmany (1990) were derived for the LMC they are probably not the most suitable values to apply to the SMC. Yet given the lack of any calibration for the SMC and the errors involved in these calibrations we find they are the only appropriate choice.

The stellar radius was determined using a method described by Kudritzki (1980)

$5 \log R_{\star}=29.58-\left(M_{v}-V\right)$

where,

$V=-2.5 \log \int_{0}^{\infty} F_{\lambda} S_{v}^{0}(\lambda) \mathrm{d} \lambda$

$F_{\lambda}$ is the stellar flux from the final theoretical model of each star (units: $\operatorname{erg~s}^{-1} \mathrm{~cm}^{-2} \AA^{-1}$ ) and $S_{v}^{0}(\lambda)$ is the $V$-filter response function for air mass zero from Matthews \& Sandage (1963). The stellar luminosities and masses were then calculated using the effective temperatures, gravities, and radii determined from our analysis.

The error on the stellar radius is dominated by the uncertainty in our distance estimate. The distance to the SMC has been determined from many different astrophysical objects (viz. Cepheids, eclipsing binaries and RC stars) and has been reviewed thoroughly in Harries et al. (2003). From the various estimates the SMC lies at a DM between 18.7-19.1, therefore we adopt an error of \pm 0.2 . With an additional error of 0.1 from the adopted apparent magnitudes, this infers an error of \pm 0.22 on the absolute magnitude estimates giving a $10 \%$ uncertainty in the stellar radii. This uncertainty in the radius introduces larger errors in the luminosity and mass estimates, on the order of $15 \%$ and $30 \%$, respectively. A systematic error is also introduced to the derived mass-loss rates due to the uncertainty in the adopted distance to the SMC, this is discussed further in Sect. 4.2.2.

\subsection{Wind parameters}

In addition to the stellar parameters which describe their photospheric conditions, the spectra provide information on their stellar winds. The stellar wind is parameterised by the massloss rate $(\dot{M})$, the exponent of the velocity law ( $\beta$-parameter), and the terminal velocity $\left(v_{\infty}\right)$. The former two parameters are derived here from the $\mathrm{H}_{\alpha}$ profile and the latter from UV P-Cygni profiles.

\subsubsection{Terminal velocity, $v_{\infty}$}

Terminal velocities of AV 215, Sk 191, AV 210 and AV 18 have been presented in EVANS04, where a more detailed discussion can be found. Briefly, $v_{\infty}$ was derived from the UV P Cygni lines using the method described by Haser et al. (1995). This procedure utilises the Sobolev Exact 
Integration (SEI) method introduced by Lamers et al. (1987; see also Hamann 1981). For early B-type stars the doublets Si IV 1394, 1403 and C IV 1548, 1551 display the most prominent wind features and thus were the diagnostic lines used by EVANS04, see the fits to these profiles in Fig. 2 of EVANS04.

Unfortunately four stars in our sample showed no significant wind features in the aforementioned UV lines; AV 104, AV 216, AV 362 and AV 22 (EVANS04). Therefore terminal velocities for these stars were estimated by an alternative method. Radiation driven wind theory (Castor et al. 1975; Abbott 1978) predicts that terminal and escape velocities scale together as a function of $\alpha$, such that $v_{\infty} / v_{\text {esc }} \sim \hat{\alpha} /(1-\hat{\alpha})$, if the finite cone-angle subtended by the stellar disk is accounted for. $\hat{\alpha}$ is dependent on the effective temperature and is defined as the effective value of the force multiplier parameter, $\alpha$, defining the exponent of the line strength distribution function. Lamers et al. (1995) found, for stars with temperatures $\geq 21 \mathrm{kK}$, that the $v_{\infty} / v_{\text {esc }}$ ratio was approximately 2.6 but that at lower temperatures $(<20 \mathrm{kK})$ this dropped to 1.3 . Kudritzki \& Puls (2000) found, from a comparison of the Lamers sample of 68 stars with an additional set of stars ( 200) from Prinja \& Massa (1998), that above $21 \mathrm{kK}$ this ratio is 2.65 and below $21 \mathrm{kK}$ it is 1.4 .

In EVANS04 a comparison of $v_{\infty} / v_{\text {esc }}$ derived from the Lamers et al. samples discussed above with those derived from SMC OB-type supergiants, showed no convincing variation in this relationship due to the different metallicity environments. The average $v_{\infty} / v_{\text {esc }}$ from the SMC OB-type supergiants with $T_{\text {eff }}>21 \mathrm{kK}$ is within the scatter of the Galactic star results. Therefore in order to derive the terminal velocities of AV 104, $\mathrm{AV} 216, \mathrm{AV} 362$ and $\mathrm{AV} 22$ we have adopted the $v_{\infty} / v_{\mathrm{esc}}$ ratio of 2.65 for the former two stars and 1.4 for the two latter, cooler stars. The escape velocities were derived using the same procedure as described in EVANS04 (see Eqs. (1) and (2)), where $v_{\text {esc }}$ was calculated from the ratio of stellar mass to radius and corrected for the radiative acceleration due to electron scattering. We should note that in all three samples (Lamers et al. 1995; Prinja \& Massa 1998; EVANS04) there is a large scatter of approximately $20 \%$ in the $v_{\infty} / v_{\text {esc }}$ at a given temperature. This is reflected in the the error estimates for $v_{\infty}$ given in Table 4 for the four stars where we have used this method.

\subsubsection{Mass-loss rate and $\beta$-parameter}

The mass-loss rate is derived mainly from the morphology of the $\mathrm{H}_{\alpha}$ profile, with less weight given to $\mathrm{H}_{\beta}$ profile. This procedure has been described in detail in Puls et al. (1996) and Kudritzki et al. (1999). The $\beta$-parameter effects the central emission core of the $\mathrm{H}_{\alpha}$ profile and also its FWHM. Therefore for thick winds (i.e. when there is emission in the $\mathrm{H}_{\alpha}$ profile), the $\beta$-parameter and mass-loss rate can be obtained simultaneously. The derived mass-loss rates are presented in Table 4, the uncertainties quoted are the random errors involved in the fitting process which are typically in the range 10-15\%. In addition we should consider the systematic error incurred through the adopted distance modulus. The uncertainty in DM follows through to the mass-loss rate from the $10 \%$ uncertainty it induces on the stellar radius. If we assume that we get identical profiles as long as $Q$ is constant (i.e. that we are fitting $Q$ and not $\dot{M})$, where $Q=\dot{M} / R_{\star}^{3 / 2}$, then the systematic error in the derived mass-loss rates is $\pm 15 \%$ (Puls et al. 1996). Fits to the $\mathrm{H}_{\alpha}$ profiles are presented in Fig. 3, where we show the best fits (red line) along with models representing a change in $\dot{M}$ of $\pm 15 \%$ (green and blue lines). Some nebular contamination was detected in Sk 191, AV 362 and AV 22 from comparisons between the UVES and EMMI datasets. After careful extraction of the nebular contribution to the $\mathrm{H}_{\alpha}$ profiles the EMMI data were considered more reliable for AV 362 and AV 22 whilst for Sk 191 the UVES data appeared less contaminated. In fitting the $\mathrm{H}_{\alpha}$ line of these stars any obvious nebular component was avoided (see Fig. 3).

For those stars with thin winds, (AV 104 and AV 216), $\mathrm{H}_{\beta}$ was used as a constraint on the $\beta$-parameter, whilst the core depth of the $\mathrm{H}_{\alpha}$ line constrained $\dot{M}$ (see Fig. 3). For too high a $\beta$-parameter, an emission peak appears in the $\mathrm{H}_{\beta}$ theoretical profile which is not observed. This method provides an upper limit for the $\beta$-parameter.

In the case of AV 215 (BNO Ia), the profile shape of the $\mathrm{H}_{\alpha}$ line provided a means of determining both $\dot{M}$ and $\beta$ simultaneously. However, the models could not simultaneously reproduce the depth and width of the absorption core. Kudritzki et al. (1999) noticed this problem in a B1 Iab-type supergiant, HD 13854, suggesting it may be a lack of line-blanketing which was affecting the stratification of the source function of $\mathrm{H}_{\alpha}$ throughout the wind. This is not supported by our analysis as it has been carried out with a version of FASTWIND that includes an approximation of the line-blanketing and blocking. This inability to reproduce the depth of the blueward absorption core is also seen for AV 18 (B2 Ia). This star is similar to the B2 Ia type stars HD 41117 and HD 14143 discussed by Kudritzki et al. (1999), in that its emission peak could be fitted giving $\dot{M}$ and $\beta$, whilst the absorption core could not be matched.

The $\mathrm{H}_{\alpha}$ profiles of the two B1.5 Ia type stars illustrate the extreme differences between the winds of stars with the same spectral and luminosity classification; whilst Sk 191 is in emission, AV 210 is in absorption. In Fig. 3 we show two fits to AV 210 , one with $\beta=1$ and the other with $\beta=3$. Although, these are extremely different profiles the mass-loss rate only varies by $25 \%$. We have adopted the parameters from the $\beta=3$ case as the observed $\mathrm{H}_{\beta}$ line is reproduced and the $\mathrm{H}_{\alpha}$ absorption core depth, though not the profile shape, can also be matched.

In the coolest stars, AV 362 and AV 22, $\dot{M}$ and $\beta$ were derived from the height and width of the $\mathrm{H}_{\alpha}$ emission. Note in Fig. 3 that the code predicts large absorption troughs not observed in the actual data, yet they are an inevitable consequence of the Lyman continuum and the Lyman resonance line becoming optically thick at lower temperatures and large mass-loss rates (e.g. Kudritzki \& Puls 2000). Although there is probably some nebular contamination in the $\mathrm{H}_{\alpha}$ profile of both stars, it is unlikely to have masked such a large absorption core. One can speculate that this problem might be resolved by including the effects of clumping in the analysis, since the massloss rates derived here would be overestimated and the Lyman 
Table 5. Derived non-LTE absolute abundances for SMC B-type supergiants. Abundances are given as $\log [N(\mathrm{X}) / N(\mathrm{H})]+12$, where $\mathrm{X}$ represents the appropriate element. Numbers in the parenthesis represent the number of lines used in the abundance analysis. The errors represent the standard deviation of the mean and account for systematic errors as discussed in Sect. 5.

\begin{tabular}{|c|c|c|c|c|c|c|c|}
\hline$\overline{\text { Star }}$ & 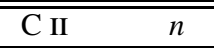 & $\overline{\mathrm{N} \text { II }}$ & $\overline{\mathrm{O} \text { II }}$ & Mg II & $\overline{\overline{\text { Si II }}}$ & 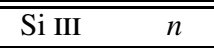 & S Si IV \\
\hline AV 215 & $6.91 \pm 0.10(1)$ & $7.96 \pm 0.12(3)$ & $7.97 \pm 0.14$ & $<7.20$ & & $7.10 \pm 0.15(3)$ & $7.14 \pm 0.12(1)$ \\
\hline AV 104 & $<7.00$ & $7.41 \pm 0.15$ & $8.05 \pm 0.32(12)$ & $6.90 \pm 0.05(1)$ & & $6.70 \pm 0.11$ & $6.67 \pm 0.22(1)$ \\
\hline AV 216 & $6.76 \pm 0.16(1)$ & $7.14 \pm 0.20(1)$ & $8.22 \pm 0.39$ & $6.64 \pm 0.08$ & & $6.66 \pm 0.22(3)$ & $<6.71$ \\
\hline Sk 191 & $6.89 \pm 0.10$ & $7.63 \pm 0.19(6)$ & $8.20 \pm 0.18(12)$ & $6.98 \pm 0.06(1)$ & & $6.75 \pm 0.16(3)$ & $6.50 \pm 0.25$ \\
\hline AV 210 & $6.93 \pm 0.14$ & $7.60 \pm 0.12(7)$ & $8.18 \pm 0.18(12)$ & $6.89 \pm 0.07(1)$ & & $6.69 \pm 0.14$ & $6.70 \pm 0.11$ \\
\hline AV 18 & $6.98 \pm 0.18$ & $7.50 \pm 0.30(7)$ & $8.26 \pm 0.21(12)$ & $6.75 \pm 0.10$ & $<7.03$ & $6.81 \pm 0.11(3)$ & \\
\hline AV 362 & $7.12 \pm 0.48$ & $8.22 \pm 0.25$ & & $6.72 \pm 0.06(1)$ & $6.60 \pm 0.13(2)$ & $6.66 \pm 0.19(3)$ & \\
\hline AV 22 & $7.07 \pm 0.27(3)$ & $7.92 \pm 0.16$ & & $6.83 \pm 0.09(1)$ & $6.75 \pm 0.16(2)$ & $6.61 \pm 0.15(2)$ & \\
\hline
\end{tabular}

optical depths might be reduced. We will come back to this again in Sect. 6.3 however a detailed discussion of the effects of clumping is beyond the scope of this work.

\section{Chemical abundance analysis}

In previous investigations of the atmospheres of supergiants, there has been clear evidence of chemical processing (McErlean et al. 1999). The photospheres of these luminous stars have chemical patterns which indicate contamination of the stellar surface by the products of $\mathrm{CN}$-cycle burning and thus we have carried out an abundance analysis on this stellar sample to study this further. Adopting the atmospheric parameters presented in the previous section we derived the chemical composition of our stellar sample. The results of the absolute abundance analysis are presented in Table 5. We have also compared our mean abundances to those derived for B-type giants and main-sequence stars in NGC 330, an unprocessed B-type star AV 304 and SMC H II regions, see Table 6.

The most dominant uncertainty on B-type supergiant abundances is the adopted microturbulence. As discussed in Sect. 4.1.3 each element implies a different value for the microturbulence (see Table 3). As we have adopted the microturbulence from the $\mathrm{Si}$ III lines, $\xi_{\mathrm{Si}}$, it is necessary to explore the effect of this choice of microturbulence on the other elements. Firstly the uncertainty in $\xi_{\mathrm{Si}}$ is $\pm 2 \mathrm{~km} \mathrm{~s}^{-1}$, which introduces an error of $\sim 0.05$ for weak lines and typically \pm 0.10 for strong lines. For example the carbon abundance, which depends mainly on the weak C II feature at $4267 \AA$ only varies by \pm 0.02 for the B0-B1.5 stars. As this line becomes stronger for the B2-B5 -type stars the effect of microturbulence is larger increasing to \pm 0.07 . The largest effects of the uncertainty in $\xi_{\mathrm{Si}}$ are on the abundances of Si IV in AV 215 and AV 104 and nitrogen in AV 362 and AV 22. If we adopt the microturbulence from the oxygen lines, there is a decrease in the abundance of carbon, nitrogen, and magnesium of normally less than 0.1 dex. However the silicon and oxygen abundances are decreased by up to 0.2 dex depending on the degree of change in the microturbulence and the strength of the spectral features.

\subsection{Helium}

Achieving a consistent fit to both the singlet and triplet neutral helium lines in a stellar spectrum is a known problem.
Voels et al. (1989) concluded that the extension of the stellar atmosphere was causing this effect and could be resolved by the inclusion of sphericity in the model atmospheres. Studies of OB-type supergiants which omit microturbulence tend to find very high helium fractions, of up to 0.4 , in order to get a fit to all He I lines (Lennon et al. 1991; Herrero et al. 1992). By including a non-zero microturbulence McErlean et al. (1998) found that a more consistent, although not perfect, fit to the helium lines could be achieved with a solar or near solar helium fraction of 0.09 . Due to the difficulty in constraining the helium abundance from the neutral helium lines we have adopted a solar helium fraction of 0.09 . No significant variation from this helium abundance was noted in our sample, although consistent fits to the helium spectra were not always achieved. We should note that the helium abundance has an indirect effect on the temperature and gravity and hence the mass-loss rate. McErlean et al. (1998) showed that by changing the helium fraction from 0.1 to 0.2 , the $T_{\text {eff }}$ and $\log g$ were decreased by $0.5 \mathrm{kK}$ and 0.05 dex, respectively, which would change $\dot{M}$ by less than $10 \%$.

\subsection{Carbon, nitrogen and oxygen}

The carbon abundances are based primarily on the C II $4267 \AA$ line, with the $6580 \AA$ multiplet being visible for the B2-B5 stars (see Fig. 3). From Table 5 we see that the mean carbon abundance, from those stars for which it is derived solely from the $4267 \AA$ line (B0-B1.5), is \pm 0.1 dex lower than that determined by including the $6580 \AA$ multiplet (B2-B5). In the coolest stars the $6580 \AA$ multiplet actually gives abundances of $\sim 0.25$ dex higher than those determined from the $4267 \AA$ line. The $4267 \AA$ line is known to be particularly sensitive to non-LTE effects (Eber \& Butler 1988), resulting in lower abundances than derived from other carbon lines (viz. 3919, 3921, $4650 \AA$ ). This has been found by LEN03 in the non-LTE analyses of B-type stars in NGC 330 and that of an unprocessed B-type star AV 304 (LEN03; Rolleston et al. 2003). However the carbon abundances for the stars in our sample show little scatter and have a mean abundance for the sample of 6.96 dex.

The mean oxygen abundance is in agreement with these previous studies, the large errors in Table 5 represent the uncertainty caused by adopting $\xi_{\mathrm{Si}}$. No oxygen abundance could 
Table 6. Comparison of mean abundances for the SMC B-type supergiants with previous studies of abundances in the SMC. Along with our mean B-type supergiant abundances as derived from Table 5 we show the mean abundances of A-type supergiants from Venn et al. (1999, 2003b; Si corrected for non-LTE effects.), SMC main-sequence B-type star AV 304 (Rolleston et al. 2003; corrected for non-LTE effects), NGC 330 cluster main-sequence B-type stars (LEN03; corrected for non-LTE effects), and SMC H II regions from Kurt et al. (1999). The errors represent the standard deviation of the mean.

\begin{tabular}{lccccc}
\hline \hline & \multicolumn{2}{c}{ Supergiants } & \multicolumn{2}{c}{ B stars } & H II \\
Metal & This work & A-type & NGC 330 & AV 304 & regions \\
\hline $\mathrm{C}$ & $7.30 \pm 0.09$ & & $7.26 \pm 0.15$ & $7.41 \pm 0.18$ & $7.53 \pm 0.06$ \\
$\mathrm{~N}$ & $7.67 \pm 0.27$ & $7.52 \pm 0.10$ & $7.51 \pm 0.18$ & $6.55 \pm 0.01$ & $6.59 \pm 0.08$ \\
$\mathrm{O}$ & $8.15 \pm 0.07$ & $8.14 \pm 0.06$ & $7.98 \pm 0.13$ & $8.16 \pm 0.33$ & $8.05 \pm 0.05$ \\
$\mathrm{Mg}$ & $6.78 \pm 0.16$ & $6.83 \pm 0.08$ & $6.59 \pm 0.14$ & 6.73 & \\
$\mathrm{Si}$ & $6.74 \pm 0.11$ & $6.92 \pm 0.15$ & $6.58 \pm 0.32$ & $6.74 \pm 0.03$ & $6.70 \pm 0.20$ \\
\hline
\end{tabular}

be determined for the AV 362 and $\mathrm{AV} 22$, as these are too cool for the presence of visible oxygen lines in their spectra.

The nitrogen abundances for our sample exhibit a large scatter with the mean being $7.67 \mathrm{dex}$, a factor of 13 more overabundant than AV 304.

\section{3. $\alpha$-process elements; magnesium and silicon}

Magnesium abundances are based solely on the Mg II $4481 \AA$ doublet but are reasonably consistent throughout our sample. For AV 215, the magnesium line was weak and blended with a He I line, thus providing us with only an upper limit.

The silicon abundance is very sensitive to the adopted temperature and this is clear from the range of abundances presented in Table 5. However, the mean abundance agrees well with those of AV 304 and SMC H II regions.

\section{Discussion}

\subsection{Chemical composition of SMC}

Before analysing our samples composition, it is useful to discuss the baseline composition of the SMC to which we can relate our absolute abundances. Rolleston et al. (2003) showed that the main-sequence star AV 304 has a chemical composition similar to that derived from SMC H II regions. Since this star shows no signs of processing in its photosphere it should represent the present-day composition of the SMC. However this was an LTE analysis and in order to compare our non-LTE abundances with those of AV 304 we need to take into account the non-LTE effects. LEN03 carried out such corrections on AV 304 and their NGC 330 B-type star sample. In addition they applied corrections to the carbon abundance to account for the lower abundances derived from the $4267 \AA$ line. As mentioned in Sect. 5.2 the absolute carbon abundance is effected by the problematic line $4267 \AA$, which gives abundances lower than those derived by weaker $\mathrm{C}$ II lines. Thus a direct comparison of the carbon abundance derived from $4267 \AA$ and that from H II regions is inappropriate. Gies \& Lambert (1992) and Vrancken et al. (2000), derived carbon abundances from weak lines and the $4267 \AA$ line, respectively. From a comparison of these studies LEN03 found the weak lines gave abundances which were
0.34 dex higher than those determined from the $4267 \AA$ line and subsequently applied this correction to the carbon abundances of AV 304 and the NGC 330 stars. We have also applied this correction to our derived carbon abundances which rely mainly on the $4267 \AA$ Aine. In Table 6 we present the corrected AV 304 abundances that can be used as a baseline SMC chemical composition along with the SMC H II region abundances (Rolleston et al. 2003; Kurt et al. 1999). Note the excellent agreement between AV 304 and the $\mathrm{H}$ II regions.

Also included in Table 6 are the mean abundances from our B-type supergiant sample, from SMC A-type supergiants (Venn et al. 1999, 2003b) and the corrected abundances of NGC 330 B-type stars (LEN03). The Si abundance presented in Venn et al. (1999) was derived from LTE calculations, corrections similar to those carried out for AV 304 and the NGC 330 stars have been applied to this value to account for non-LTE effects. Comparing the abundances of the $\alpha$-processed elements ( $\mathrm{Mg}$ and $\mathrm{Si}$ ) in each set of objects we see, as expected, that they are in reasonable agreement indicating no processing of these elements. In fact assuming that the galactic standards of LEN03 and Rolleston et al. (2003) represent the galactic B-type star abundances, we find that the $\alpha$-processed elements in our sample are 0.42 dex lower than the galactic objects. This is in agreement with the 0.44 dex difference found between HR 2387 and AV 304 (Rolleston et al. 2003).

The most significant result is the variation in the nitrogen abundances. Whilst the AV 304 nitrogen abundance and that of the $\mathrm{H}$ II regions agree, those for the $\mathrm{B}$ and A-type supergiants and the NGC 330 stars are a factor of 9 to 14 greater. It is interesting to note that within the B-type supergiant sample itself, a sizeable variation in nitrogen abundance is also present (see Table 5). These enhancements are much greater than any error incurred in our analysis and are therefore considered to be real. The most likely explanation for such an enhancement of nitrogen is that we are seeing the CNO processed material contaminating the chemical composition of the photosphere. If this is the case, with such high nitrogen abundances we might expect a corresponding but smaller enhancement in helium with depletions of carbon and possibly oxygen, keeping the sum of CNO nuclei constant. However a comparison of the oxygen abundance with that of AV 304 shows excellent agreement, with no indication of an underabundance present. On the other hand 
carbon appears to be underabundant by 0.11 and 0.23 in comparison to AV 304 and the $\mathrm{HII}$ regions, respectively. This is comparable to the carbon depletions produced in the Maeder \& Meynet (2001) stellar evolution models from similar nitrogen enhancements $(<0.25 \mathrm{dex})$. Nevertheless given the uncertainties in the carbon abundance and the magnitude of the correction applied to carbon due to the $4267 \AA$ line, this slight underabundance may not be significant. The CNO pattern of our B-type supergiant sample is in good agreement with that of the A-type supergiants and NGC 330 B-type stars i.e. N is enhanced, whilst $\mathrm{C}$ and $\mathrm{O}$ appear to be of normal SMC metallicity.

\subsection{Evolutionary status}

To help understand the evolutionary status of these B-type supergiants/giant we have compared our results to the stellar evolution tracks of Maeder \& Meynet (2001) which include rotationally induced mixing (see Figs. 4, 6 and 7). As noted by LEN03 the initial abundances of these models are scaled to solar abundances and so by adopting $0.2 Z_{\odot}$ they greatly overestimate the initial nitrogen abundance i.e. instead of an SMC nitrogen abundance of 6.55, they use one-fifth solar or 7.3 dex. We have recalibrated these models to SMC nitrogen abundances, following the discussion of LEN03, who assumed the initial nitrogen abundance has no effect on the amount of excess nitrogen produced and simply rescaled it to the SMC metallicity.

The position of our stellar sample on the HR-diagram is shown in Fig. 4, overlayed on the rotating (- -) and non-rotating (-) tracks of Maeder \& Meynet (2001). Note that all our stars lie redward of the main-sequence phase, with the two coolest stars having positions consistent with the core-helium burning phase (this phase is represented by the bump at $\log T_{\text {eff }}=$ 4.2-4.3). By interpolating between the rotating stellar evolution tracks we estimated the evolutionary masses $\left(M_{\mathrm{evol}}\right)$ of the sample, which are listed in Table 3. Figure 5 illustrates the difference between $M_{\text {evol }}$ and the derived spectroscopic masses, $M_{\text {spec }}$, which were determined from the stellar radii and surface gravities (see Table 3). This plot implies a mass discrepancy where $M_{\text {evol }}>M_{\text {spec }}$, for most of the sample. LEN03 suggested that this mass discrepancy, could be accounted for by the uncertainties in the surface gravity which were considerably larger in their work (due to low $S / N$ ). Moreover the spectroscopic masses are dependent on the distance modulus (DM) which introduces an additional systematic error. In Fig. 5 the solid and dashed line represents the zero point of $\log \left(M_{\mathrm{evol}} / M_{\mathrm{spec}}\right)$ and the errors on this value due to the adopted DM. The errors in the gravity and distance modulus can account for some of this mass discrepancy but there remains an inconsistency of 0.1-0.2 dex. We note that one of our stars, AV 216, has $M_{\text {evol }}<M_{\text {spec }}$, similar to one star from LEN03. As discussed in Sect. 4.1.2 we may have overestimated the surface gravity of this star due to the presence of a companion and this would, in turn, lead to an overestimation of $M_{\text {spec }}$.

In Fig. 6 we plot the recalibrated stellar evolution tracks, showing the development of the surface nitrogen abundances as

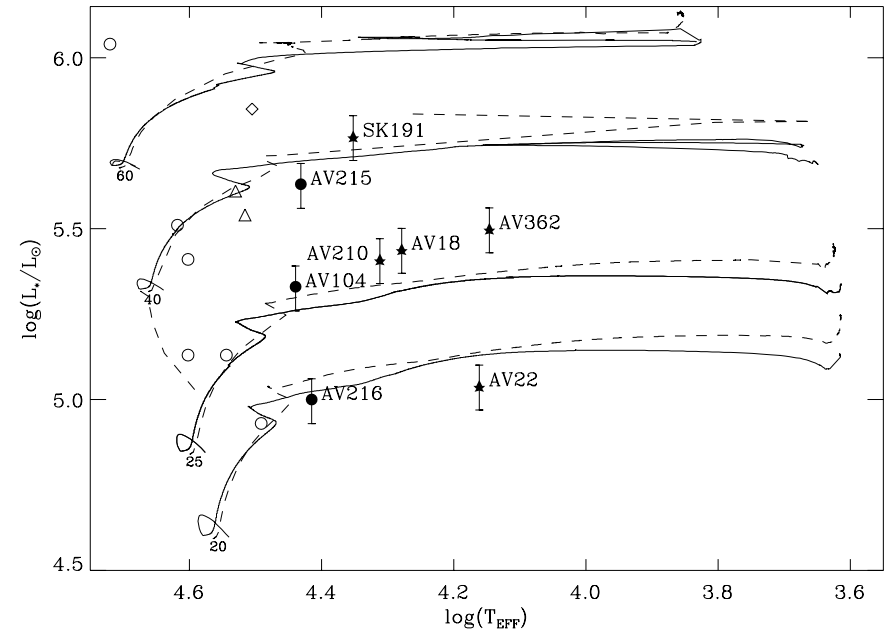

Fig. 4. HR-diagram, luminosity as a function of temperature. Shown are the stellar evolution tracks of Maeder \& Meynet (2001) for an assumed initial rotational velocity of 0 (-; solid lines) and $300 \mathrm{~km} \mathrm{~s}^{-1}$ (- -; dashed lines) at stellar masses of 20, 25, 40 and $60 M_{\odot}$. Included are the results from our early and mid B-type supergiants $(\bullet ; \star)$, with errors in luminosity representing $\pm 15 \%$, SMC O-type supergiants of Hillier et al. $(\triangle ; 2003)$ and Crowther et al. $(\diamond ; 2002)$, and the SMC O-type dwarfs of Bouret et al. (o; 2003).

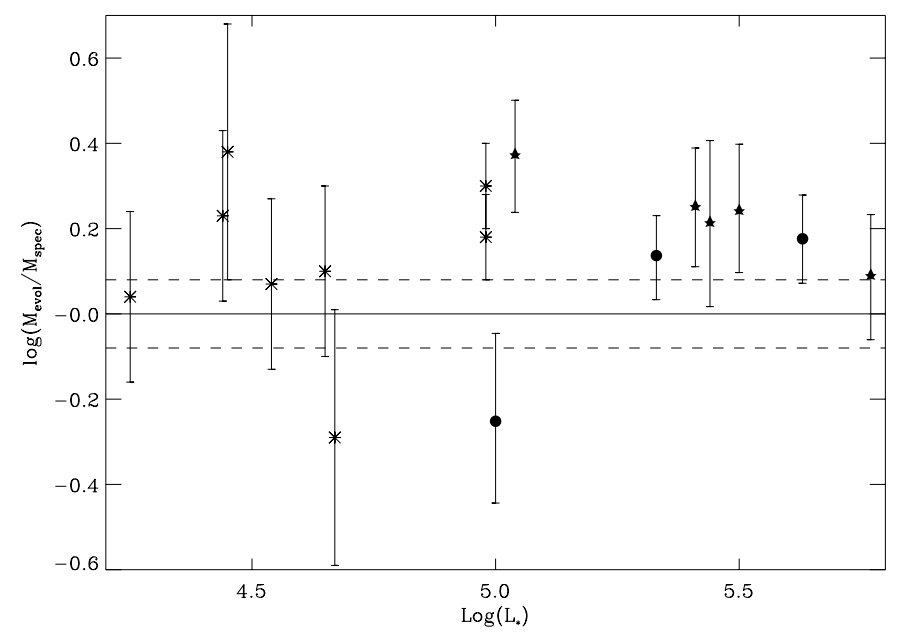

Fig. 5. Comparison of evolutionary and spectroscopic masses as a function of luminosity. Included are the results from our early and mid B-type supergiants $(\bullet ; \star)$ and NGC 330 B-type stars from Lennon et al. $(* ; 2003)$. The error bars represent the error in the surface gravity. Also shown is the uncertainty in mass due to the adopted distance modulus (- -).

a function of temperature. We have included the photospheric nitrogen abundances of all the SMC OBA-type supergiants and dwarfs available in the literature (Hillier et al. 2003; Crowther et al. 2002; Bouret et al. 2003; LEN03; Venn et al. 2003b) as well as the results from our B-type supergiants. The initial rotational velocity of the stellar models which can reproduce nitrogen abundances similar to those observed needs to be at least $300 \mathrm{~km} \mathrm{~s}^{-1}$ and these models show no significant slowdown by the end of the hydrogen-burning stage. Although these rapidly rotating models imply significant nitrogen enhancements some of the OBA-type supergiants have abundances up to a factor of five larger than predicted. 


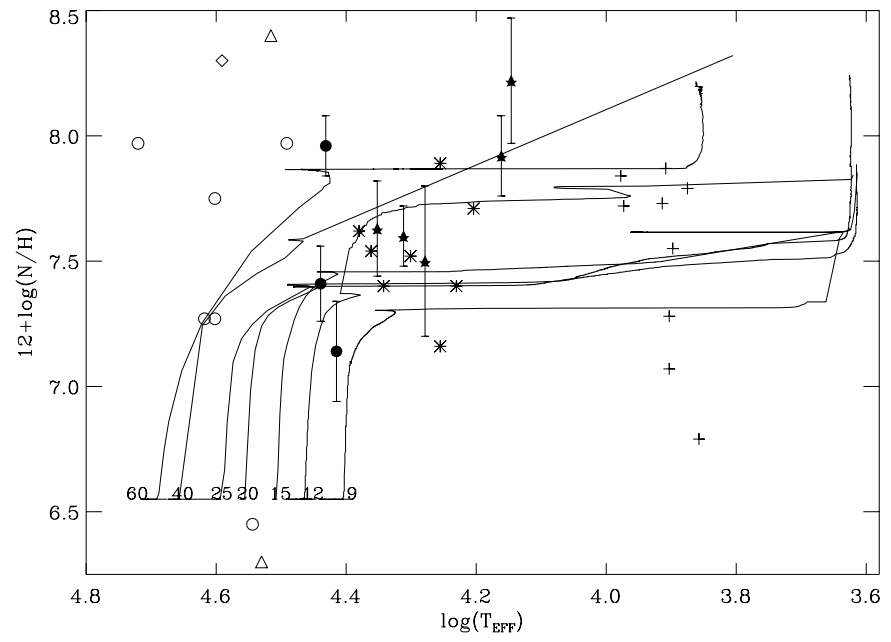

Fig. 6. Surface nitrogen abundances as a function of temperature. Shown are the stellar evolution tracks of Maeder \& Meynet (2001) for an assumed initial rotational velocity of $300 \mathrm{~km} \mathrm{~s}^{-1}$ and various stellar masses. Included are the results from our early and mid B-type supergiants $(\bullet ; \star)$, SMC O-type supergiants of Hillier et al. $(\triangle ; 2003)$ and Crowther et al. $(\diamond ; 2002)$, SMC O-type dwarfs of Bouret et al. (०; 2003), NGC 330 B-type stars of Lennon et al. (*; 2003), and A-type supergiants from Venn et al. (+; 2003b). The error bars illustrate the random and systematic errors on the nitrogen abundance.

Figure 7 shows the observed projected rotational velocities of the OBA-type supergiants and dwarfs against the predicted rotational velocities. Note that we are probably overestimating the values for the supergiants due to spectral line broadening being dominated by macroturbulence (see Sect. 2; Howarth et al. 1997; Ryans et al. 2002). The observed velocities are significantly lower than expected from the stellar evolution models. In order for these stars to have rotational velocities similar to those predicted, the inclination of the stellar rotation axis would have to be $<20^{\circ}$. Assuming that the distribution of the rotational axis, $i$, is random, it is highly unlikely that our sample of stars have such large rotational velocities and that we are observing them all pole on.

The O-type dwarfs of Bouret et al. (2003) have masses similar to the masses of our sample, as such we will consider them to be representative of the progenitors for the B-type supergiants. Since these O-type stars show a similar range of nitrogen enhancements to the B-type supergiants, it is reasonable to assume that the additional nitrogen is produced while the stars are still on the main-sequence. If the nitrogen enhancement is truly a product of rotationally induced mixing, this process needs to be more efficient at lower rotational velocities than predicted by Maeder \& Meynet (2001), see also the discussion of Herrero \& Lennon (2002).

A factor that needs to be carefully considered in stellar evolution models is the effect of the mass-loss rate. This is incorporated in the Maeder \& Meynet (2001) models and is responsible for the loss of angular-momentum through the stellar wind. These models use mass-loss rates from Kudritzki \& Puls (2000) and are modified by rotation using an expression dependent on $\alpha$, a force multiplier parameter. Maeder \& Meynet assume that $\alpha=0.6$ but as discussed in Sect. 6.3 this quantity is not

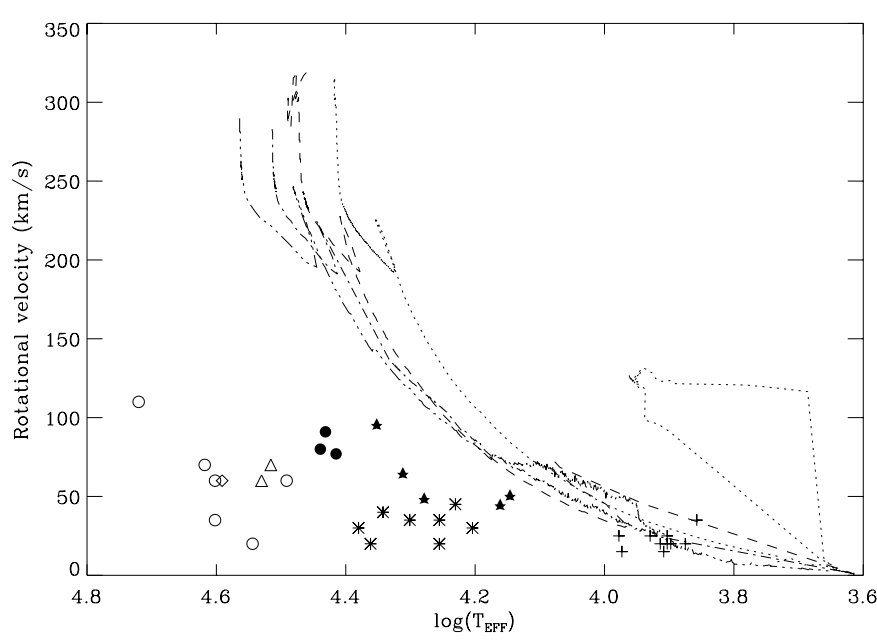

Fig. 7. Rotational velocities as a function of temperature. Shown are the stellar evolution tracks of Maeder \& Meynet (2001) for an assumed initial rotational velocity of $300 \mathrm{~km} \mathrm{~s}^{-1}$ and various stellar masses. Included are the $v \sin i$ values from our early and mid B-type supergiants $(\bullet ; \star)$, SMC O-type supergiants of Hillier et al. $(\triangle ; 2003)$ and Crowther et al. $(\diamond ; 2002)$, SMC O-type dwarfs of Bouret et al. (०; 2003), NGC 330 B-type stars Lennon et al. (*; 2003), and A-type supergiants from Venn et al. (+; 1999).

reliably established for B-type stars. Additionally Kudritzki \& Puls (2000) used only unblanketed analyses of the O and B type supergiants, whilst more recent analyses will give more accurate mass-loss rates due to their inclusion of line-blanketing in the model atmosphere codes (see Sect. 6.3 for a further discussion). Clearly some allowance for the variation of mass-loss rates as a function of metallicity should also be included in the stellar evolution models.

Binary star evolution models by Wellstein et al. (2001), can also produce nitrogen enriched blue supergiants with similar temperatures and luminosities to the SMC supergiants in our sample. Through mass transfer from the primary star, the low mass secondary in a binary system becomes more massive. These stars may appear to have lower spectroscopic masses than expected from their luminosity. Thus this could account for some of the mass discrepancy in our stellar sample. One advantage of the binary evolution models, is that they can place these blue supergiants on the post main-sequence gap which presently can not be achieved with single-star models due to their rapid evolution through this part of the HR-diagram. Since the primary of the binary system will evolve into a low mass helium star by the time the secondary is a blue supergiant, it is unlikely that the binary systems could be detected through radial velocity variations. Wellstein et al. (2001) predict radial velocity variations of $\sim 15 \mathrm{~km} \mathrm{~s}^{-1}$.

\subsection{Winds of B-type supergiants}

According to radiative line-driven wind theory, the windmomentum and hence mass-loss rate should be dependent on metallicity and may be expressed as a function of the force multiplier parameter, $\alpha$. This parameter is the ratio of the optically thick line acceleration to the total line acceleration 
and the metallicity dependence of the mass-loss rate is found to scale as

$\dot{M} \sim\left(Z_{*} / Z_{\odot}\right)^{\frac{1-\alpha}{\alpha^{\prime}}}$

where, $\alpha^{\prime}=\alpha-\delta$ and $\delta$ is a force multiplier parameter that accounts for the ionisation balance in the wind (Kudritzki et al. 1989; Puls et al. 2000). Theoretically, this metallicity dependence is predicted to be in the range $Z^{0.5}-Z^{0.85}$ for OB-type supergiants (Kudritzki et al. 1987; Leitherer et al. 1992; Vink et al. 2001). Observationally, a significant enough sample of stars has not yet been analysed to get a reliable estimate of $\alpha$. However for three SMC O-type supergiants Puls et al. (1996) derived an exponent of 0.52, whilst for 6 O-type dwarfs analysed by Bouret et al. (2003) a value of 0.6 was determined. Unfortunately due to the dependence of mass-loss on the stellar luminosity and mass, a comparison of the known Galactic B-type supergiant mass-loss rates and those derived here will not depend solely on their different metallicities.

Vink et al. (2001) provide a recipe to determine the massloss rate which includes a metallicity dependence of 0.85 . Three different formulae are used to describe the stellar massloss depending on its position relative to the so called "bistability jumps", which vary due to the temperature and density regime of the star. In Fig. 8 we compare our observed mass-loss rates with these theoretical predictions. Note that for the early B-type stars (B0-B1) Vink's predictions, which are based on the recipe for stars above the first jump, underestimates $\dot{M}$ by a factor of 4, whilst for the mid B-type stars the recipe for between the two bistability jumps overestimates $\dot{M}$ by a factor of 10 . This underestimation for the early B-type supergiants has also been observed for O-type supergiants and dwarfs by Bouret et al. (2003) and Evans et al. (2003). If we use the steeper exponent of 0.5 for the metallicity dependence in Vink's recipes we get good agreement with the mass-loss rates for the early B-type supergiants but the rates for the mid B-type supergiants are now over estimated by a factor of 16 . Clearly, a better understanding of the metallicity and temperature dependence of mass-loss rates for B-type supergiants is required.

An alternative perspective is provided by considering the wind momentum, $D_{\text {mom }}=\left(\dot{M} v_{\infty} R^{1 / 2}\right)$. As described in the introduction, radiative line-driven wind theory relates the windmomentum of a star to its luminosity. In recent years massive star analyses have endeavoured to calibrate this relationship as a function of spectral type and metallicity, with the plan to invoke it as a method of deriving distances from OBA-type supergiants out to the Virgo and Fornax clusters (Puls et al. 1996; Kudritzki et al. 1999, 2000; Crowther et al. 2002; Bouret et al. 2003; Bresolin et al. 2001; Urbaneja et al. 2003; Evans et al. 2003). In Fig. 9 we compare the wind-momenta of the SMC Btype supergiants/giant studied here with the Galactic analysis of Kudritzki et al. (1999). The solid lines in this figure represent the Galactic WLR relationships as derived from the recent lineblanketed O-type star analysis of Repolust et al. (2003) and the unblanketed early and mid B-type supergiant analyses of Kudritzki et al. (1999, 2000). Surprisingly, the SMC stars from B0-B2 agree well with the galactic relationship. However the

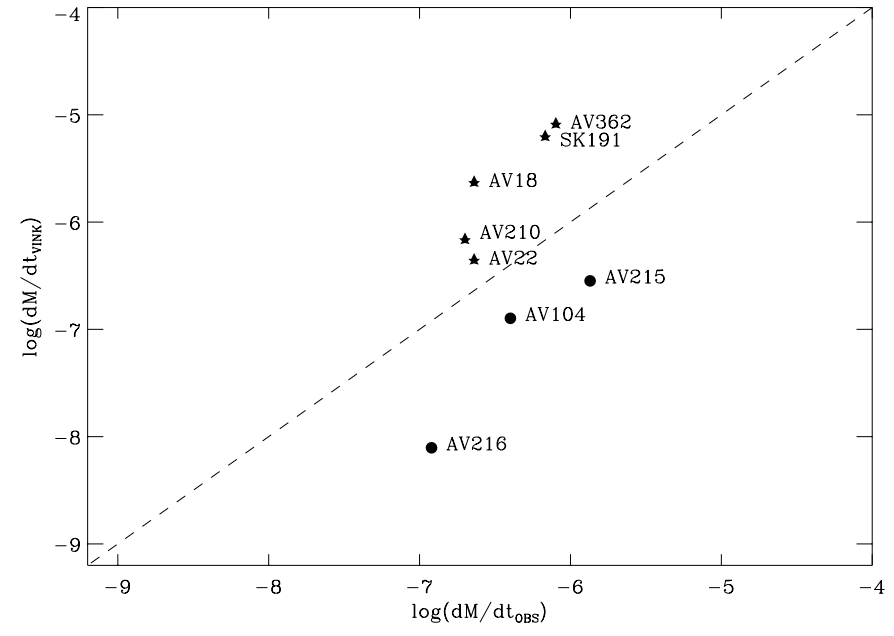

Fig. 8. Comparison of theoretical and observed mass-loss rates. Theoretical predictions are calculated using the metallicity dependent mass-loss recipes of Vink et al. (2001) for $Z=0.2 Z_{\odot}$. The solid circles $(\bullet)$ represent the SMC early B-type supergiants, whilst the solid stars $(\star)$ represent the mid B-type supergiants.

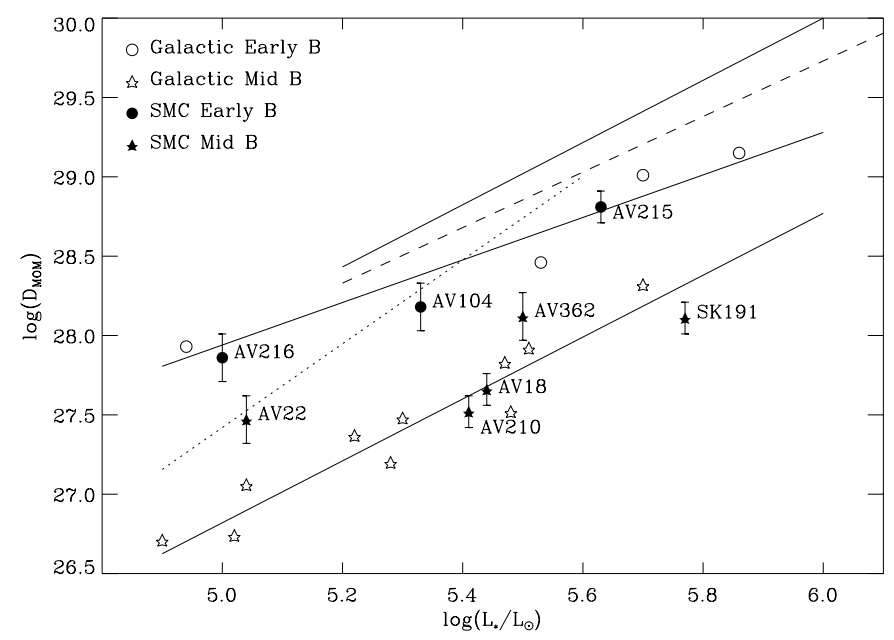

Fig. 9. Wind momentum as a function of Luminosity. The solid lines are the wind-momentum-luminosity relationship, in decreasing values of wind momentum, from galactic O-type supergiants without clumping and with line-blanketing (Repolust et al. 2003) and from galactic early and mid B-type supergiants (Kudritzki et al. 1999). The dashed line (- - ) represents the unblanketed WLR for O-type supergiants determined by Puls et al. (1996), whilst the dotted line $(\cdots)$ represents that for the A-type supergiants determined by Kudritzki et al. (1999). Note the galactic BA-type WLR's were calibrated using an unblanketed version of FASTWIND. The plot shows the early and mid B-type stars of the galaxy (o, unfilled stars ; Kudritzki et al. 1999) and the $\operatorname{SMC}(\bullet, \star$; this work $)$.

two cool stars (B3-B5) have wind-momenta which lie above the mid $B$ relationship, this is more significant in the case of the B5 star, AV 22. We must note that the mid B WLR of Kudritzki et al. (1999) was based on spectral types no later than B3, with the coolest star in their sample, HD 14134, being at $18 \mathrm{kK}$ in comparison to the $14 \mathrm{kK}$ of AV 362. From Fig. 9 we see that AV 22 agrees well with the WLR calibrated by Kudritzki et al. (1999) for A-type stars. It is plausible that this cooler spectral type should follow the A-type star WLR or indeed a different calibration of the WLR for late B-type 

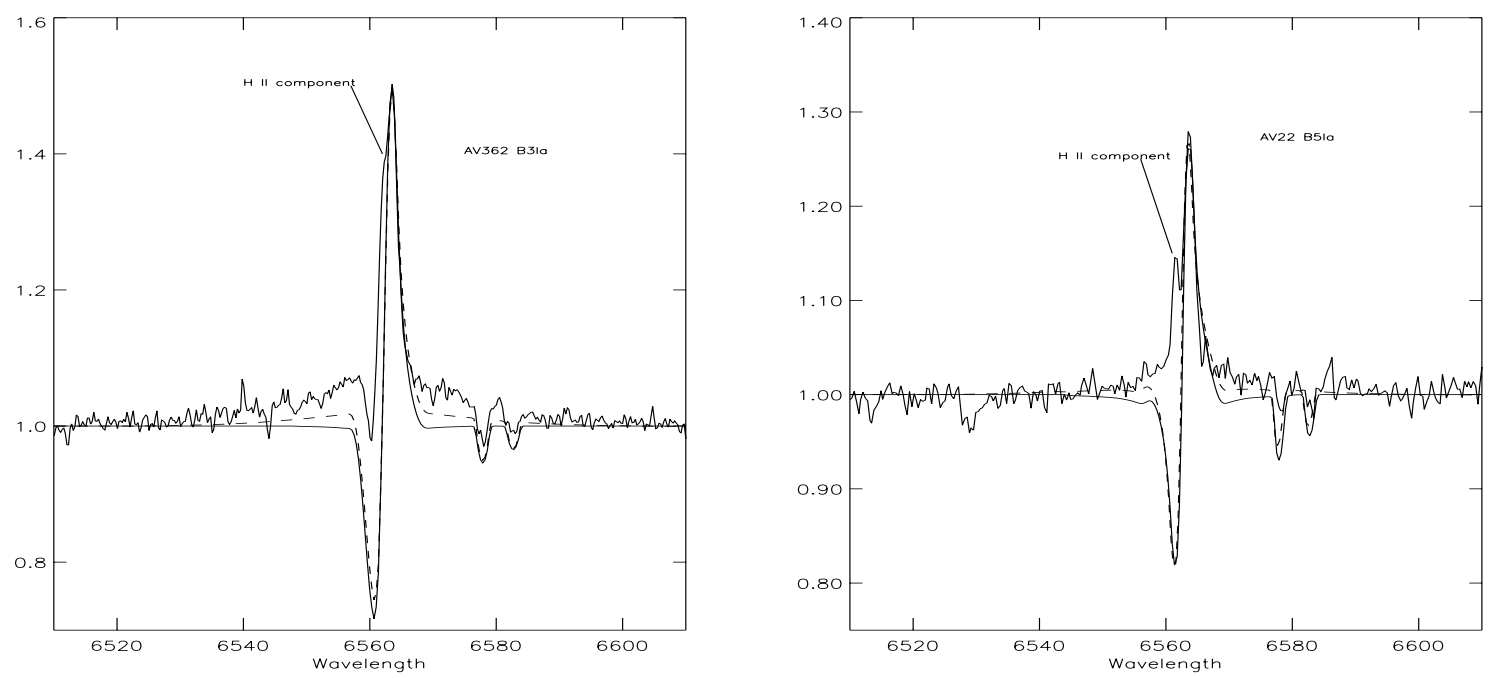

Fig. 10. To show the effect of incoherent electron scattering on the wings of AV 362 and AV 22. Shown are the best fits (solid line; —-) using the parameters presented in Tables 3 and 4 and the same model with the inclusion of electron scattering (dashed line; - - -). Note the improvement in the fit to the wings of AV 22 by including electron scattering and the little effect the scattering has on the fit to AV 362.

stars. Yet without more stars of similar or cooler spectral types (B5-B9), we can not make any definite conclusions. Moreover the inclusion of clumping into our analysis would decrease the mass-loss rate in such a way as to bring the wind-momenta of AV 362 and AV 22 into better agreement with the mid B WLR.

From Fig. 3 it is apparent that the (far) wings of $\mathrm{H}_{\alpha}$ in these stars are quite strong and are unaccounted for by the FASTWIND models. In Fig. 10 we show the results of including incoherent electron scattering in the FASTWIND calculations. The electron scattering only increases the strength of the continuum around the $\mathrm{H}_{\alpha}$ by $2 \%$ and $1 \%$ in AV 362 and AV 22, respectively, where as the observed spectrum shows a raise in the $\mathrm{H}_{\alpha}$ wings of $7 \%$ and $3 \%$. Whilst the situation has improved for AV 22, the electron scattering does little to improve the fit to $\mathrm{H}_{\alpha}$ in $\mathrm{AV} 362$. Note that the fact that the electron-scattering can not account for the full strength of the electron scattering wings most probably is not related to any presence or nonpresence of wind-clumping, since these wings are formed in the upper photosphere (cf. Kudritzki et al. 1999).

The agreement of our analysis with the galactic WLR for early B-type stars maybe somewhat misleading as the analysis of Kudritzki et al. (1999) was undertaken using an unblanketed version of FASTWIND. Repolust et al. (2003) and Puls et al. (1996) have carried out a line-blanketed and unblanketed analysis, respectively, of a set of Galactic O stars. In Fig. 9 the regressions fits of both analyses are plotted and imply that the line-blanketed version of the O-type star WLR is steeper. Additionally for a given luminosity, higher wind-momenta are required than for the unblanketed results. Since a similar effect on the WLR is expected for the B-type stars, it is therefore possible that this is masking any predicted metallicity effect. In addition the uncertainty in the adopted distance to the SMC introduces a systematic error to the derived radii and mass-loss rates, this will also effect the wind-momentum luminosity relationship. This changes the WLR through the wind-momentum and the luminosity, shifting the relationship along both the horizontal and vertical directions of Fig. 9.
In general, the fitting uncertainties on the mass-loss rates are of the order $10-15 \%$, however the assumption of no clumping in our models introduces an additional uncertainty. Crowther et al. (2002) and Evans et al. (2003) noticed some indication of clumping in their O- and B-type supergiants from observations in the UV and far UV (see also Massa et al. 2003; Hillier et al. 2003). Moreover Repolust et al. (2003) suggested the presence of clumping to explain certain inconsistencies between observed and theoretically predicted WLR's. Since the mass-loss rate varies with the filling factor as $\dot{M} / \sqrt{ } f$, assuming a filling factor of 0.1 reduces the mass-loss to one-third of its value before considering clumping. Although clumping is an important factor to be considered in the analysis of supergiants with thick winds, the degree of clumping is still somewhat uncertain. Whilst for WR stars the clumping enhances the strength of the coherent electron scattered wings of the lines, this effect (which must not be confused with the incoherent electron scattering wings discussed above) is too small for detection in the OB-type supergiants (see Hillier et al. 2003). In addition by including clumping into the analyses of massive stars the mass-loss rate will be reduced and hence we would expect a shift in the WLR to lower wind-momenta (Repolust et al. 2003).

\section{Conclusions}

From high-resolution UVES data we have derived the atmospheric and wind parameters of seven SMC B-type supergiants and one giant in the spectral range B0-B5. In addition we have carried out an abundance analysis on each of these stars. This analysis was undertaken using the non-LTE line-blanketed atmospheric code FASTWIND, which through spherical symmetry and extended atmospheres allows for the computation of the stellar wind (Santoloya-Rey et al. 1997; Herrero et al. 2002; Repolust et al. 2003).

The absolute abundances of the $\alpha$-processed elements $(\mathrm{O}$, $\mathrm{Mg}$ and $\mathrm{Si}$ ) and carbon in these SMC stars are in good 
agreement with SMC H II regions and an unprocessed B-type star, AV 304 (Kurt et al. 1999; Rolleston et al. 2003). The exception is nitrogen which in the giant, AV 216, is enhanced by a factor of four while there is a mean overabundance of a factor of 13 present in the supergiants. Slightly lower nitrogen enhancements are observed in B-type giants in NGC 330 and A-type supergiants (Lennon et al. 2003; Venn et al. 1999).

A comparison was carried out with the most recent stellar evolution models of Maeder \& Meynet (2001) which include rotationally induced mixing. However we recalibrated these models to an SMC initial nitrogen abundance of $6.55 \mathrm{dex}$ as observed in AV 304 instead of the one-fifth solar implemented by Maeder \& Meynet. These models can for the most part reproduce the observed nitrogen abundances if an initial rotational velocity of $300 \mathrm{~km} \mathrm{~s}^{-1}$ is invoked. Nevertheless, observed projected rotational velocities are $<100 \mathrm{~km} \mathrm{~s}^{-1}$ and therefore we conclude that these models need to have more efficient mixing at lower initial rotational velocities. In addition we note that the evolutionary masses are generally higher than the derived spectroscopic masses by at least a factor of 1.2. Some of this discrepancy may be accounted for in the errors of the adopted distance modulus and possibly the gravity, leaving a residual difference in masses of less than 0.1 dex where $M_{\text {evol }}>M_{\text {spec }}$.

The theoretical mass-loss rate predictions of Vink et al. (2001) which implement a metallicity dependence of $Z^{0.64}$, underestimate $\dot{M}$ for the early B-type stars and overestimate it for the mid B-types. By using a steeper metallicity dependence $\left(Z^{0.5}\right)$, the early B-type stars are in agreement but the situation is then worse for the later spectral types. Clearly the metallicity dependence of mass-loss rates for B-type stars is still uncertain and a comparison of equivalent sets of stars in different metallicity environments is needed to understand this dependence.

Finally, we have compared our results to the windmomentum relationships of Galactic B-type stars by Kudritzki et al. (1999, 2000). The wind momenta of our stars are in good agreement with the galactic B-type supergiants. This is a puzzling result given the theoretical predictions of a metallicity dependence on the wind-momenta and the observational evidence of this effect for O-type supergiants in the Magellanic Clouds (Puls et al. 1996; Bouret et al. 2003). However the observed Galactic WLR was derived using an unblanketed analysis which may mask any predicted metallicity dependence when compared to our results.

Work is currently underway to analyse EMMI spectra of additional SMC early B-type supergiants in the spectral range B0-B2.5. This larger sample of stars will hopefully provide more insight into the behaviour of the wind-momenta and stellar evolution of these early B-type supergiants at low metallicities.

Acknowledgements. C.T. is grateful to the Department of Higher and Further Education, Training and Employment for Northern Ireland (DEFHTE) and the Dunville Scholarships fund for their financial support. D.J.L. acknowledges funding from the UK Particle Physics and Astronomy Research Council (PPARC under the grant PPA/G/S/2001/00131. We would like to thank Robert Ryans for his continuous support with TLUSTY. Based on observations collected at the European Southern Observatory, Chile (Paranal).

\section{References}

Abbott, D. C. 1978, ApJ, 225, 893

Azzopardi, M., \& Vigneau, J. 1982, A\&AS, 50, 291

Becker, S. R., \& Butler, K. 1988, A\&A, 201, 232

Becker, S. R., \& Butler, K. 1989, A\&A, 209, 244

Becker, S. R., \& Butler, K. 1990, A\&A, 235, 326

Bouret, J.-C., Lanz, T., Hillier, D. J., et al. 2003, ApJ, submitted

Bresolin, F., Kudritzki, R.-P., Mendez, R. H., \& Przybilla, N. 2001, ApJ, 548, L159

Bresolin, F., Gieren, W., Kudritzki, R.-P., Pietrzynski, G., \& Przybilla, N. 2002a, ApJ, 567, L277

Bresolin, F., Kudritzki, R.-P., Najarro, F., Gieren, W., \& Pietrzynski, G. 2002b, ApJ, 577, 107

Butler, K., \& Giddings, J. R. 1985, Coll. Comp. Project No. 7 (CCP7), Newsletter 9, London, 7

Castor, J. I., Abbott, D. C., \& Klein, R. I. 1975, ApJ, 195, 157

Crowther, P. A., Hillier, D. J., Evans, C. J., et al. 2002, ApJ, 579, 774

de Mello, D. F., Leitherer, C., \& Heckman, T. M. 2000, ApJ, 530, 251

Denissenkov, P. 1994, A\&A, 287, 113

Dufton, P. L., McErlean, N. D., Lennon, D. J., \& Ryans, R. S. I. 2000, A\&A, 353, 311

Eber, F., \& Butler, K. 1988, A\&A, 202, 153

Eggenberger, P., Meynet, G., \& Maeder, A. 2002, A\&A , 386, 576

Evans, C. J., Crowther, P. A., Fullerton, A. W., \& Hillier, D. J. 2003, MNRAS, submitted

Evans, C. J., Lennon, D. J., Trundle, C., Heap, S. R., \& Lindler, D. J. 2004, ApJ, submitted

Fitzpatrick, E. L., \& Bohannan, B. 1993, ApJ, 404, 734

Fitzpatrick, E. L., \& Garmany, C. D. 1990, ApJ, 363, 119

Garmany, C. D., Conti, P. S., \& Massey, P. 1987, AJ, 93, 1070

Gies, D. R., \& Lambert, D. L. 1992, ApJ, 387, 673

Gray, D. F. 1992, The observation and analysis of stellar photospheres (Cambridge University Press), 2nd ed.

Hamann, W.-R. 1981, A\&A, 93, 353

Harries, T. J., Hilditch, R. W., \& Howarth, I. D. 2003, MNRAS, 339, 157

Haser, S. M., Lennon, D. J., Kudritzki, R.-P., et al. 1995, A\&A, 295, 136

Haser, S. M., Pauldrach, A. W. A., Lennon, D. J., et al. 1998, A\&A, 330,285

Heger, A., \& Langer, N. 2000, ApJ, 544, 1016

Herrero, A., Kudritzki, R.-P., Vilchez, J. M., et al. 1992, A\&A, 261, 209

Herrero, A., Puls, J., \& Najarro, F. 2002, A\&A, 396, 949

Herrero, A., \& Lennon, D. J. 2002, Stellar rotation, IAU Symp., 215, in press

Hillier, D. J., \& Miller, D. L. 1998, ApJ, 496, 407

Hillier, D. J., Lanz, T., Heap, S. R., et al. 2003, ApJ, 588, 1039

Howarth, I. D., Siebert, K. W., Hussain, G. A. J., \& Prinja, R. A. 1997, MNRAS, 284, 265

Howarth, I. D., \& Smith, K. C. 2001, MNRAS, 327,353

Hubeny, I., \& Lanz, T. 1995, ApJ, 439, 875

Kamp, L. W. 1978, ApJS, 36, 143

Kilian, J., Becker, S. R., Gehren, T., \& Nissen, P. E. 1991, A\&A, 244, 419

Kudritzki, R.-P. 1980, A\&A, 85, 174

Kudritzki, R.-P., Pauldrach, A. W. A., \& Puls, J. 1987, A\&A, 173, 293

Kudritzki, R.-P., Pauldrach, A. W. A., Puls, J., \& Abbot, D. C. 1989, A\&A, 219, 205

Kudritzki, R.-P. 1992, A\&A, 266, 395

Kudritzki, R.-P., Lennon, D. J., \& Puls, J. 1995, in Science with the VLT, ed. J. R. Walsh, \& I. J. Danziger (Heidelberg: Springer), 246

Kudritzki, R.-P., Puls, J., Lennon, D. J., et al. 1999, A\&A, 350, 970 
Kudritzki, R.-P., \& Puls, J. 2000, ARA\&A, 38, 613

Kurt, C. M., Dufour, R. J., Garnett, D. R., et al. 1999, ApJ, 518, 246

Lamers, H. J. G. L. M., Cerruti-Sola, M., \& Perinotto, M. 1987, ApJ, 314,726

Lamers, H. J. G. L. M., Snow, T. P., \& Lindholm, D. M. 1995, ApJ, 455,269

Langer, N., \& Maeder, A. 1995, A\&A, 295, 685

Leitherer, C., Robert, C., \& Drissen, L. 1992, ApJ, 401, 596

Leitherer, C., Leão, J. R. S., Heckman, T. M., et al.2001, ApJ, 550, 724

Lennon, D. J., Brown, P. L. F., Dufton, P. L., \& Lynas-Gray, A. E. 1986, MNRAS, 222, 719

Lennon, D. J., Kudritzki, R.-P., Becker, S. T., et al. 1991, A\&A, 252, 498

Lennon, D. J., Dufton, P. L., Mazzali, P. A., Pasian, F., \& Marconi, G. 1996, A\&A, 314, 243

Lennon, D. J. 1997, A\&A, 317, 87

Lennon, D. J., Dufton, P. L., \& Crowley, C. 2003, A\&A, 398, 455

Lyubimkov, L. S. 1991, in Evolution of stars: the photospheric abundance connection, ed. G. Michaud, \& A. V. Tutukov (Dordrecht: Kluwer), IAU Symp., 145, 125

Maeder, A., \& Meynet, G. 2000, A\&A, 361, 159

Maeder, A., \& Meynet, G. 2001, A\&A, 373, 555

Martins, F., Schaerer, D., \& Hillier, D. J. 2002, A\&A, 382, 999

Massa, D., Fullerton, A. W., Sonneborn, G., \& Hutchings, J. B. 2003, ApJ, 586, 996

Massey, P. 2002, ApJS, 141, 81

Matthews, T. A., \& Sandage, A. R. 1963, ApJ, 138, 30

McErlean, N. D., Lennon, D. J., \& Dufton, P. L. 1998, A\&A, 329, 613

McErlean, N. D., Lennon, D. J., \& Dufton, P. L. 1999, A\&A, 349, 553

Mihalas, D. 1972, ApJ, 177, 115

Monteverde, M. I., Herrero, A., Lennon, D. J., \& Kudritzki, R.-P. 1996, A\&A, 312, 24

Monteverde, M. I., Herrero, A., Lennon, D. J., \& Kudritzki, R.-P. 1997, ApJ, 474L, 107

Monteverde, M. I., \& Herrero, A. 1998, Ap\&SS, 263, 171

Monteverde, M. I., Herrero, A., \& Lennon, D. J. 2000, ApJ, 545, 813

Osmer, P. S. 1972, ApJ, 171, 393

Osmer, P. S. 1973, ApJ, 184, 127

Pauldrach, A. W. A., Hoffmann, T. L., \& Lennon, M. 2001, A\&A, 375, 161

Pettini, M., Steidel, C. C., Adelberger, K. L., Dickinson, M., \& Giavalisco, M. 2000, ApJ, 528, 96

Prinja, R. K., \& Massa, D. L. 1998, in Properties of Hot Luminous Stars, ed. I. Howarth (San Francisco: ASP), ASP Conf. Ser., 131, 218
Przybylski, A. 1968, MNRAS, 139, 313

Przybylski, A. 1971, MNRAS, 152, 197

Puls, J., Kudritzki, R.-P., Herrero, A., et al. 1996, A\&A, 305, 171

Puls, J., Springmann, U., \& Lennon, M. 2000, A\&AS, 141, 23

Repolust, T., Puls, J., \& Herrero, A. 2003, A\&A, submitted

Rolleston, W. R. J., Venn, K., Tolstoy, E., \& Dufton, P. L. 2003, A\&A, 400, 21

Rucinski, S. M. 1990, PASP, 102, 306

Ryans, R. S. I. R., Dufton, P. L., Rolleston, W. R. J., et al. 2002, MNRAS, 336, 577

Santolaya-Rey, A. E., Puls, J., \& Herrero, A. 1997, A\&A, 323, 488

Sanduleak, N. 1968, AJ, 73, 246

Schaller, G., Schaerer, D., Meynet, G., \& Maeder, A. 1992, A\&AS, 96, 269

Smartt, S. J., Crowther, P. A., Dufton, P. L., et al. 2001, MNRAS, 325, 257

Talon, S., Zahn, J. P., Maeder, A., \& Meynet, G. 1997, A\&A, 322, 209

Trundle, C., Dufton, P. L., Lennon, D. J., Smartt, S. J., \& Urbaneja, M. A. 2002, A\&A, 395, 519

Urbaneja, M. A., Herrero, A., Bresolin, F., et al. 2003, ApJ, 584, L73

Vazquez, G. A., Leitherer, C., Heckman, T. M., et al. 2003, ApJ, submitted

Venn, K. A. 1995, ApJ, 99, 659

Venn, K. A. 1999, ApJ, 518, 405

Venn, K. A., McCarthy, J. K., Lennon, D. J., et al. 2000, ApJ, 541, 610

Venn, K. A., Lennon, D. J., Kaufer, A., et al. 2001, ApJ, 547, 765

Venn, K. A., Tolstoy, E., Kaufer, A., et al. 2003a, AJ, 126, 1326s

Venn, K. A., \& Przybilla, N. 2003b, in CNO in the Universe, ed. C. Charbonnelm, D. Schaerer, \& G. Meynet (San Francisco: ASP), ASP Conf. Ser., 304, in press [astro-ph/0212258]

Vink, J. S., deKoter, A., \& Lamers, H. J. G. L. M. 2001, A\&A, 369, 574

Voels, S. A., Bohannan, B., Abbott, D. C., \& Hummer, D. G. 1989, ApJ, 340, 1073

Vrancken, M. 1998, Ph.D. Thesis, Vrije Universiteit Brussel

Vrancken, M., Lennon, D. J., Dufton, P. L., \& Lambert, D. L. 2000, A\&A, 358, 639

Walborn, N. R., Lennon, D. J., Haser, S. M., Kudritzki, R.-P., \& Voels, S. A. 1995, PASP, 107, 104

Walborn, N. R., Lennon, D. J., Heap, S. R., et al. 2000, PASP, 112, 1243

Wellstein, S., Langer, N., \& Braun, H. 2001, A\&A, 369, 939

Wolf, B. 1972, A\&A, 20, 275

Wolf, B. 1973, A\&A, 28, 335 